Journal for ImmunoTherapy of Cancer

\title{
Metformin reduces PD-L1 on tumor cells and enhances the anti-tumor immune response generated by vaccine immunotherapy
}

\author{
Luis Enrique Munoz, ${ }^{1}$ Lei Huang, ${ }^{1}$ Ramireddy Bommireddy, ${ }^{1}$ Richa Sharma, ${ }^{2}$ \\ Lenore Monterroza, ${ }^{1}$ Rohini N. Guin (1) , ${ }^{1}$ Sarah G. Samaranayake, ${ }^{1}$ \\ Christopher D. Pack, ${ }^{3}$ Sampath Ramachandiran, ${ }^{3}$ Shaker J.C. Reddy, ${ }^{3}$ \\ Mala Shanmugam, ${ }^{2}$ Periasamy Selvaraj (i) ${ }^{1}$
}

To cite: Munoz LE, Huang L, Bommireddy R, et al. Metformin reduces $\mathrm{PD}-\mathrm{L} 1$ on tumor cells and enhances the anti-tumor immune response generated by vaccine immunotherapy. Journal for ImmunoTherapy of Cancer 2021;9:e002614. doi:10.1136/ jitc-2021-002614

- Additional supplemental material is published online only. To view, please visit the journal online (http://dx.doi.org/10. 1136/jitc-2021-002614).

Accepted 15 October 202

Check for updates

(c) Author(s) (or their employer(s)) 2022. Re-use permitted under CC BY-NC. No commercial re-use. See rights and permissions. Published by BMJ.

${ }^{1}$ Pathology and Laboratory Medicine, Emory University, Atlanta, Georgia, USA ${ }^{2}$ Hematology and Medical Oncology, Emory University, Atlanta, Georgia, USA

${ }^{3}$ Metaclipse Therapeutics Corporation, Atlanta, Georgia, USA

Correspondence to Professor Periasamy Selvaraj; pselvar@emory.edu

\section{ABSTRACT}

Background PD-L1 is one of the major immune checkpoints which limits the effectiveness of antitumor immunity. Blockade of PD-L1/PD-1 has been a major improvement in the treatment of certain cancers, however, the response rate to checkpoint blockade remains low suggesting a need for new therapies. Metformin has emerged as a potential new drug for the treatment of cancer due to its effects on PD-L1 expression, $T$ cell responses, and the immunosuppressive environment within tumors. While the benefits of metformin in combination with checkpoint blockade have been reported in animal models, little remains known about its effect on other types of immunotherapy.

Methods Vaccine immunotherapy and metformin were administered to mice inoculated with tumors to investigate the effect of metformin and TMV vaccine on tumor growth, metastasis, PD-L1 expression, immune cell infiltration, and CD8 T cell phenotype. The effect of metformin on IFN- $\gamma$ induced PD-L1 expression in tumor cells was assessed by flow cytometry, western blot, and RT-qPCR.

Results We observed that tumors that respond to metformin and vaccine immunotherapy combination show a reduction in surface PD-L1 expression compared with tumor models that do not respond to metformin. In vitro assays showed that the effect of metformin on tumor cell PD-L1 expression was mediated in part by AMP-activated protein kinase signaling. Vaccination results in increased $T$ cell infiltration in all tumor models, and this was not further enhanced by metformin. However, we observed an increased number of CD8 T cells expressing PD-1, Ki-67, Tim-3, and CD62L as well as increased effector cytokine production after treatment with metformin and tumor membrane vesicle vaccine.

Conclusions Our data suggest that metformin can synergize with vaccine immunotherapy to augment the antitumor response through tumor-intrinsic mechanisms and also alter the phenotype and function of CD8 T cells within the tumor, which could provide insights for its use in the clinic.

\section{INTRODUCTION}

The major roadblocks in the development of immunotherapy approaches for cancer are tumor-induced immunosuppression and the lack of an effective antitumor immune response. ${ }^{1} 2$ Immune suppression within tumors can be mediated by many factors, including expression of checkpoint molecules, secreted factors, and a lack of nutrients and resources for immune cells. Immune checkpoint inhibitors (ICIs) that target PD-L1 and CTLA-4 are at the forefront for clinical interventions. ${ }^{34}$ The blockade of the PD-1/PD-L1 axis using antibodies has been successful in the clinic for the treatment of malignant diseases that were previously deemed untreatable. Despite these advances in treatment, response rates to ICI therapy remain low ${ }^{5}$ and often rely on the pre-existing endogenous immune response that may not be present in all patients. ${ }^{67}$

We have developed a vaccine immunotherapy approach that uses tumor membrane vesicles (TMVs) derived from tumors as an antigen source. ${ }^{8-10}$ These TMVs are incorporated with immunostimulatory molecules (ISMs), such as interleukin (IL) 12 and B7-1, using a novel protein transfer platform that uses these ISMs modified with a glycosylphosphatidylinositol (GPI) tail so they can be anchored onto the surface of TMVs to generate the TMV vaccine. ${ }^{9-11}$ TMVs containing GPIISMs have been shown to induce immunity in murine thymoma, ${ }^{11}$ breast cancer ${ }^{9}{ }^{12}$ and head and neck cancer. ${ }^{13}$ Since these TMV vaccines contain the unique antigenic signature of each individual tumor, the platform provides an opportunity to deliver a personalized vaccine immunotherapy.

In recent years, metformin has emerged as a potential therapeutic agent for cancer therapy. ${ }^{14}$ Metformin is a biguanide that can act through AMP-activated protein kinase (AMPK)-dependent and independent mechanisms, inhibiting mitochondrial respiration 
and resulting in decreased gluconeogenesis in patients with diabetes. ${ }^{15}$ It has been shown to have direct and indirect antitumor properties, such as the ability to modulate PD-L1 in the tumor, ${ }^{16}{ }^{17}$ and the metabolic remodeling of tumors. ${ }^{18}$ While metformin has been shown to synergize with immune checkpoint blockade in preclinical models, it is not known if combination with vaccine immunotherapy can alter the vaccine efficacy.

Here, we show that metformin augments TMV vaccine immunotherapy only in tumor models which downregulates PD-L1 expression on metformin treatment. This modulation of PD-L1 was observed in tumors that could signal through AMPK after metformin treatment. Further, we observed that the metformin-mediated reduction in PD-L1 occurs on tumor cells, but not on dendritic cells (DCs), macrophages, or myeloid-derived suppressor cells (MDSCs) in the tumor. We also found changes in tumor-infiltrating CD8 T cell phenotype and their ability to produce inflammatory cytokines after TMV vaccine and metformin treatment, suggesting that metformin acts via tumor intrinsic and extrinsic mechanisms leading to therapeutic benefit in reducing primary tumor growth and metastatic burden.

\section{MATERIALS AND METHODS Animals}

$\mathrm{BALB} / \mathrm{c}$ and C57BL/ 6 mice (female, $6-8$ weeks old) were obtained from Jackson Laboratories and housed in the Division of Animal Resources (DAR) facilities. Female mice were selected to match the sex of origin from 4T1, murine oral carcinoma-1 (MOC1) and murine oral carcinoma-2 (MOC2) cell lines and to avoid rejection or the influence on vaccine-induced immunity.

\section{Reagents and cell lines}

4T1 (CRL-2539) was obtained from ATCC, CMT-167 (a metastatic clone of CMT 64) was obtained from MilliporeSigma. MOC1 and MOC2 cell lines were kind gifts from Dr Ravindra Uppaluri (Dana-Farber Cancer Institute, Harvard University). To generate PD-L1 KO 4T1, cells received a Cas9 RNP (Thermo Scientific) with a gRNA targeting PD-L1 or control RNA (PD-L1: CAGCTTGTCCAACTGGTCGG, Thermo Scientific). Seven days after transfection, 4T1 PD-L1 KO cells were

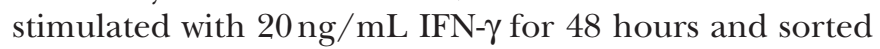
by FACS on PD-L1 negative cells. This stimulation and sorting were repeated twice to ensure purity of the PD-L1 KO population. ${ }^{19}$ Cell lines were routinely verified using their expression profile of distinct surface markers and morphology, as well as tested for mycoplasma contamination. All cell lines were cultured and expanded in complete DMEM with $4500 \mathrm{mg} / \mathrm{L}$ glucose (MilliporeSigma) supplemented with $10 \%$ fetal bovine serum (FBS, HyClone), Glutamax (Gibco), HEPES (Gibco), and penicillin-streptomycin (MilliporeSigma). Metformin hydrochloride (Cayman Chemical) was dissolved in sterile PBS at a concentration of $250 \mathrm{mM}$, aliquoted and stored at $-20^{\circ} \mathrm{C}$. PMA/ionomycin with Brefeldin A was obtained from BioLegend. G418 and 6-Thioguanine (6-TG) were obtained from MilliporeSigma.

\section{Antibodies}

Fluorochrome-conjugated antibodies to CD45 (1:200 clone 30-F11), CD3 (1:100 clone 17A2), CD8a (1:300 clone 53-6.7), CD4 (1:300 clone GK1.5), Ly-6G (1:500 clone 1A8), CD11b (1:300 clone M1/70), Tim-3 (1:100 clone B8.2C12), PD-1 (1:100 clone 29F.1A12), F4/80 (1:100 clone BM8), PD-L1 (1:300 clone 10F.9G2), MHC class I (1:300 clones 34-1-2S and 28-8-6), MHC class II (1:300 clone M5/114.15.2), IFN- $\gamma$ (1:100 clone XMG1.2), TNF- $\alpha$ (1:100 clone MP6-XT22), and IL-2 (1:100 clone JES6-5H4) were purchased from BioLegend. The live dead fixable NIR (1:400 in PBS) was purchased from Thermo Fisher Scientific. For immunofluorescence staining, the rabbit recombinant monoclonal antibody (EPR5683) to AMPK- $\alpha 1$ (phospho T183)+AMPK- $\alpha 2$ (phospho T172) (Abcam) and Donkey F (ab') 2 anti-rabbit IgG H\&L (Alexa Fluor 594) preabsorbed (Abcam) were used. For western blot, the phospho-AMPK- $\alpha$ (Thr172) rabbit antibody (40H9, Cell Signaling Technology), the AMPK $\alpha$ rabbit antibody (D5A2, Cell Signaling Technology) and the IRDye 800CW goat antirabbit IgG (LI-COR) were used.

\section{Tumor challenge, TMV vaccination, and metformin administration}

TMV vaccines were generated from frozen tumor tissue resected from mice as previously described. ${ }^{12}{ }^{13}$ Briefly, tumor tissue was homogenized using an OmniTip homogenizer, and membranes were isolated by ultracentrifugation at $100,000 \times \mathrm{g}$ over a $41 \%$ sucrose gradient. The tumor plasma membrane vesicles at the interface were collected, washed, and stored at $-80^{\circ} \mathrm{C}$ until use. To develop the vaccine, TMVs were incorporated with purified murine GPI-anchored B7-1 and GPI-anchored IL-12 molecules (GPI-ISMs) by protein transfer. Incorporation of the GPI-ISMs into TMVs was verified by flow cytometry analysis. BALB/c mice were inoculated with $5 \times 10^{4} 4 \mathrm{~T} 1$ cells in $100 \mu \mathrm{L}$ PBS, C57BL/ 6 mice were inoculated with $5 \times 10^{5}$ CMT-167 cells, $2 \times 10^{6}$ MOC1 cells or $5 \times 10^{5}$ MOC2 cells in $100 \mu \mathrm{L}$ PBS in the hind flank subcutaneously. Metformin at $50 \mathrm{mg} / \mathrm{kg}$ in PBS was administered intraperitoneally as outlined in the figures. TMV vaccination was delivered subcutaneously on the opposite flank from the tumor inoculation site at either 100 or $200 \mu \mathrm{g}$ subcutaneously per dose for two or four doses as outlined in the figures.

\section{Lung metastasis assay}

Lungs were collected from tumor bearing mice after 25-30 days from inoculation under sterile conditions. These were then minced and digested in $1 \mathrm{mg} / \mathrm{mL}$ Collagenase IV (MilliporeSigma) for 2 hours at $37^{\circ} \mathrm{C}$ under constant motion. After digestion, the cell suspension was filtered through $70 \mu \mathrm{m}$ strainers and washed twice in selection media. Cells were resuspended in $8 \mathrm{~mL}$ of selection 
media and $1 \mathrm{~mL}$ was then plated per well for each lung digestion in six-well plates under selection media. The selection media for $4 \mathrm{~T} 1$ was complete DMEM containing 6-TG at $60 \mu \mathrm{M}$, and G418 at $800 \mu \mathrm{M}$ for CMT-167, MOC1 and MOC2. When G418 was used cells were cultured for 3 days and then transferred to medium without G418. This treatment killed lung fibroblasts without affecting tumor cells. Once one of the wells reached complete confluency (around 7-14 days depending on the model), all the wells were harvested and counted using a Cellometer T4 Auto counter (Nexelcom) and trypan blue to discriminate live and dead cells.

\section{Tumor-infiltrating lymphocytes and intracellular cytokine staining}

Tumors were harvested, weighed, minced, and digested in Liberase TL (Roche) and DNAse (Roche) for $30 \mathrm{~min}$ at $37^{\circ} \mathrm{C}$ under constant motion. Cell suspensions were filtered through $70 \mu \mathrm{m}$ strainers and washed with PBS. Total cell numbers were determined using trypan blue assay in a hematocytometer. Whole cell suspensions were then stained for surface and intracellular markers. Cells were preincubated with Live Dead NIR in PBS for $20 \mathrm{~min}$ and then with $\mathrm{Fc}$ receptor blocking antibody (Clone 2.4G2, BioLegend) in FACS buffer at $4^{\circ} \mathrm{C}$ for $10 \mathrm{~min}$ to block non-specific binding of monoclonal antibodies to immune cells. Fluorochrome-conjugated primary antibodies were added and incubated for $45 \mathrm{~min}$ with shaking at $4^{\circ} \mathrm{C}$. Cells were washed three times with FACS buffer and analyzed using BD LSRII (BD Biosciences) or Aurora Spectral Flow Cytometer (CYTEK). For experiments where cytokine production was to be measured, processing was done under sterile conditions. After obtaining single cell suspension from tumors, dead cells were removed using Annexin V-based Dead Cell Removal kit (EasySep from STEMCELL). From the live cell suspension, CD8 T cells were isolated using the EasySep Mouse CD8a Positive Selection kit II. Isolated cells were quantified and resuspended in RPMI-1640 medium containing $10 \%$ FBS with PMA/ionomycin with Brefeldin A and incubated at $37^{\circ} \mathrm{C}$ for 6 hours. Cells were then stained for surface markers as described above. Intracellular markers were stained using the eBioscience Transcription Factor Staining Buffer Set (ThermoFisher Scientific), following the two-step intracellular cytoplasmic staining protocol. Data were analyzed using FlowJo software (FlowJo).

\section{Immunofluorescence imaging of tumor sections}

Formalin-fixed paraffin-embedded (FFPE) tumors were cut into $5 \mu \mathrm{m}$ sections and mounted on SuperFrost plus glass slides ( $\mathrm{R}$ Langenbrink $\mathrm{GmbH}$ ). Tissues were dewaxed in xylene and rehydrated after serial washes with ethanol. Antigen retrieval was done in a water bath at $95^{\circ} \mathrm{C}$ with citrate buffer for $30 \mathrm{~min}$, blocking was performed with 5\% normal goat serum (MilliporeSigma). Anti-CD3 antibody [CD3-12] (Abcam) and Donkey $\mathrm{F}\left(\mathrm{ab}^{\prime}\right) 2$ antirat IgG H\&L (Alexa Fluor 488) preabsorbed (Abcam) were used to detect CD3 T cells; rabbit recombinant monoclonal antibody EPR5683 to AMPK $\alpha 1$ (phosphor T183)+AMPK $\alpha 2$ (phosphor T172) (Abcam) and Donkey F (ab') 2 antirabbit IgG H\&L (Alexa Fluor 594) preabsorbed (Abcam) were used to detect AMPK phosphorylation. DAPI (BioLegend) was used for nuclear staining and the TrueVIEW Autofluorescence Quenching Kit (Vector Laboratories) was used to reduce background and mount the slides. Samples were scanned using an Olympus IX81 inverted microscope (Olympus Life Science) using Olympus Fluoview V.4.2 software. Images were analyzed using the FIJI image processing package for ImageJ. Images were converted to eight-bit formats and used a threshold overlay to identify signals from CD3 and DAPI cells in images. These were quantified using the 'analyze particles' function and the ratio of CD3 to overall DAPI was quantified. Quantification of phospho-AMPK was done using the 'measure' function to obtain the Mean Gray Value of the p-AMPK signal per image slide.

\section{In vitro treatments of cell lines}

Cells were plated in six-well plates and allowed to settle for 8 hours, then treated with metformin at different concentrations in complete media, with or without $20 \mathrm{ng} / \mathrm{mL}$ of IFN- $\gamma$ and/or $5 \mu \mathrm{M}$ of Compound C (Dorsomorphin; from MilliporeSigma) and/or $100 \mu \mathrm{M}$ of the allosteric activator of AMPK AICAR ( $N^{1}$-( $\beta$-D-Ribofuranosyl)-5aminoimidazole-4-carboxamide; Tocris) for 24-48hours. Cell counts and viability was quantified using trypan blue, and surface markers were analyzed using flow cytometry.

\section{RNA isolation and reverse transcription quantitative real-time PCR (RT-qPCR)}

Total RNA was extracted using GeneJet RNA purification (Thermo Scientific, K0732). One $\mu g$ of total RNA was used for reverse transcription using Revert Aid first Strand cDNA Synthesis kit (Thermo Scientific, K1622) with random hexamers primers. Real-time qPCR was performed using DyNamo ColorFlash master mix (Thermo Scientific, F456L) with a 7500 Fast real-time PCR system (Applied Biosystem). For relative quantification of the PD-L1 gene (Mm03048247_m1), TaqMan Gene Expression Assay (FAM) kit (ThermoFisher) was used. The transcript levels of TBP (no-Mm01277042_m1, internal control from TaqMan) were assessed in every condition as a reference value and calculated values were normalized to control the PBS condition.

\section{Western blot analysis}

Cells were lysed using the Minute Total Protein Extraction Kit (Invent Biotechnologies). Briefly, adherent cells were rinsed twice with HBSS and kept on ice for the remainder of the process. The provided denaturing buffer was supplemented with Halt's protease and phosphatase inhibitor cocktail at $3 \times$ the recommended concentration (Thermo Fisher Scientific). Lysates were passed through the provided spin columns to remove viscosity. Following the spin, total protein concentration 
was measured using the Pierce Rapid Gold BCA protein assay kit, concentration was normalized across treatments with denaturing buffer, then proceeded to add Laemmli sample buffer (Bio-Rad) supplemented with $\beta$-mercaptoethanol. Samples were then boiled at $95^{\circ} \mathrm{C}$ for $5 \mathrm{~min}$. Lysates were separated by SDS-PAGE on Any-kD gels (Bio$\mathrm{Rad}$ ). Protein transfer to nitrocellulose membranes (LICOR) was done at $4^{\circ} \mathrm{C}$ by wet transfer in Towbin buffer (25 mM Tris, $192 \mathrm{mM}$ Glycine, $20 \% \mathrm{MeOH}(\mathrm{v} / \mathrm{v}$ ) without SDS). After transfer, the membrane was allowed to dry for 1 hour at room temperature. Blocking was done using Intercept (TBS) blocking buffer (LI-COR) for 1 hour at room temperature with gentle shaking. Primary antibodies to Thr172 p-AMPK or total AMPK- $\alpha$ (1:1000 both, Cell Signaling Technologies) were added in Intercept T20 Antibody Diluent (LI-COR) at $4^{\circ} \mathrm{C}$ overnight in a nutator. Secondary IRDye 800CW donkey antirabbit IgG antibody (1:15000 LI-COR) was added in Intercept antibody diluent for 1 hour at room temperature with gentle shaking. Membranes were analyzed using an Odyssey CLx imager (LI-COR). Each blot was acquired from an independent gel as depicted. Quantification was done using the Image Studio software from LI-COR.

\section{Statistical analysis}

All statistical analyses were performed using Prism (GraphPad). The type of statistical test, $\mathrm{p}$ value and $\mathrm{n}$ values are stated in each figure legend. In brief, tumor growth was analyzed using two-way analysis of variance (ANOVA) to assess group differences over time. Oneway ANOVA was used to analyze differences in metastatic burden in the lungs, as well as surface marker expression in vitro and ex vivo.

\section{RESULTS \\ Metformin plus TMV vaccine treatment reduces tumor growth and inhibits lung metastasis in select tumor models}

To test the efficacy of the TMV vaccine plus metformin combination across murine tumor models and genetic backgrounds, we challenged mice with four syngeneic tumor cell lines. 4T1, CMT-167, MOC1 and MOC2 cells were inoculated into mice of their respective background (BALB/c with 4T1, C57BL/6 with CMT-167, MOC1 and MOC2). In all four tumor models the TMV vaccine alone resulted in a significant decrease in tumor area (figure 1). Conversely, metformin did not provide any protection as

A
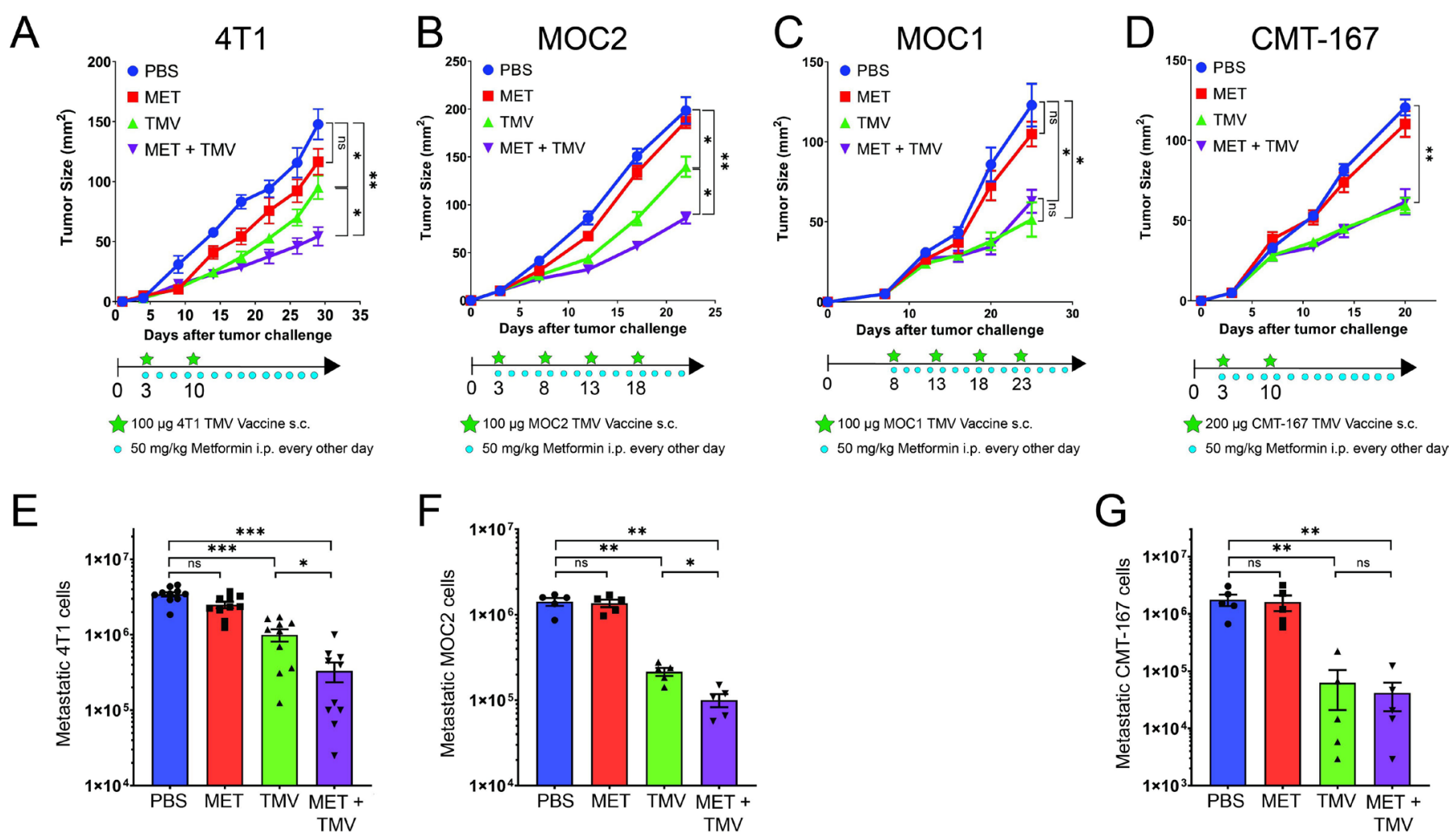
monotherapy in any of the four models. Interestingly, metformin enhanced the TMV vaccine-induced inhibition of tumor growth compared with PBS control and TMV vaccine alone (figure 1A,B). In the CMT-167 and MOC1 models no difference was observed between the combination group and TMV vaccine alone (figure $1 \mathrm{C}, \mathrm{D}$ ). We compared the growth of $4 \mathrm{~T} 1$ tumors after TMV vaccine treatment with or without PD-1 blockade or metformin. We observed that $4 \mathrm{~T} 1$ tumors are not responsive to PD-1 blockade alone, which is consistent with previously reported observations. ${ }^{20}$ However, combination with metformin and PD-1 blockade resulted in a significant reduction of tumor growth (online supplemental figure S1).

Since 4T1, CMT-167 and MOC2 tumor models are spontaneously metastatic, the lungs from tumor bearing mice were isolated to quantify metastatic cells in the lungs. The TMV vaccine was able to significantly reduce the lung metastatic burden in all the three tumor models (figure 1E-G). Metformin alone had no effect on lung metastasis as a monotherapy. The combination of TMV vaccine and metformin resulted in further reduction in lung metastatic burden in the 4T1 and MOC2 models (figure $1 \mathrm{E}, \mathrm{F}$ ), which were sensitive to metformin in combination with TMV vaccine to reduce primary tumor growth. However, lungs from CMT-167 tumor bearing mice had no difference in metastasis between TMV vaccine alone or in combination with metformin, similar to the primary tumor in this model (figure 1G).

\section{Metformin reduces tumor PD-L1 surface expression partially through TMV, AMPK signaling}

To recapitulate the inflammatory response that could be induced by the TMV vaccine in vivo and induce expression of checkpoint inhibitory molecules on tumor cells, we used IFN- $\gamma$ in vitro to determine the effect of metformin on the expression of relevant surface markers. After 48 hours of incubation with IFN- $\gamma$, PD-L1 was increased on the surface of all four cancer models although to varying degrees (figure 2A-D). While metformin failed to inhibit the IFN- $\gamma$ induced PD-L1 expression at low concentrations $(1 \mu \mathrm{M}$ to $100 \mu \mathrm{M})$, it reduced the level of IFN- $\gamma$ induced PD-L1 expression at $1 \mathrm{mM}$. Under these same conditions, the expression of the IFN- $\gamma$ receptor was not altered at any of the metformin concentrations tested (online supplemental figure S2). Importantly, the tumor models which were sensitive to the combination treatment (4T1 and MOC2) showed a significant reduction in surface PD-L1 when the cells were incubated with IFN- $\gamma$ and metformin, without affecting MHC class I (figure 2A,B). Inversely, those models that were not benefitted from combination TMV vaccine and metformin treatment (CMT-167 and MOC1) did not show a measurable change in surface PD-L1 when the cells were incubated with IFN- $\gamma$ and metformin compared with IFN- $\gamma$ alone (figure 2C,D). Interestingly, the expression of PD-L1 at the transcript level was not changed after metformin and IFN- $\gamma$ treatment, compared with IFN- $\gamma$ alone in RT-qPCR analysis of $4 \mathrm{~T} 1$ cells (online supplemental figure S3), suggesting the effect of metformin on PD-L1 is a post-transcriptional event.

Since AMPK has been reported as the pathway responsible for the reduction of PD-L1, ${ }^{16}$ we used the AMPK inhibitor Compound C (dorsomorphin) to limit AMPK signaling. In the metformin sensitive 4T1 and MOC2 cells, combination of IFN- $\gamma$ and metformin treatment resulted in decreased PD-L1 compared with IFN- $\gamma$ alone, however, when Compound $\mathrm{C}$ was added to the combination treatment, IFN- $\gamma$ induced PD-L1 was significantly elevated on the surface of both cell lines compared with IFN- $\gamma$ and metformin without Compound $\mathrm{C}$ treatment (figure 2E,F). PD-L1 remained unchanged in CMT-167 and MOC1, regardless of metformin or Compound $\mathrm{C}$ treatment (figure 2G,H). To further interrogate whether AMPK activation leads to decrease in cell surface PD-L1, cells were treated with the AMPK activator AICAR. A marked reduction of cell surface PD-L1 in the metforminresponsive MOC2 cells (figure 2I), but not in MOC1 cells was observed (figure 2J). Since AMPK phosphorylation is an indicator of AMPK activation, we investigated the phosphorylation status of AMPK both in vitro and in vivo. There was a significant increase in AMPK phosphorylation after treatment of $4 \mathrm{~T} 1$ cells with metformin in vitro, while the overall AMPK protein levels remained constant (online supplemental figure S4A,B). On 4T1 tumors resected from mice, AMPK phosphorylation was detected at a higher level in both the metformin and combination treatment groups compared with the PBS or TMV vaccine groups (online supplemental figure S4C,D).

To identify if the changes in PD-L1 were reflected in vivo after treatment with metformin and TMV vaccine administration, we measured the expression of the relevant surface markers in tumors from all four murine models. In $4 \mathrm{~T} 1$ tumors, metformin was able to significantly reduce the surface PD-L1 levels in CD45 tumor cells. While treatment of mice with the TMV vaccine resulted in an increase of surface PD-L1 on tumor cells, combination with metformin reduced surface PD-L1 levels to baseline, without any significant changes in surface MHC class I (figure 2K). In MOC2 tumors, the MHC class I levels were not changed significantly with any treatment, however, surface PD-L1 was significantly increased on TMV vaccination and decreased after combination treatment (figure 2L). In MOC1 tumors, we observed that both MHC class I and PD-L1 were significantly increased on TMV vaccination, however no effect of metformin was observed in the combination of TMV vaccine and metformin group (figure 2N). In the CMT-167 model, we observed no significant changes in the surface PD-L1 or MHC-I levels (figure 2M).

\section{Metformin does not enhance TMV vaccine efficacy in PD-L1 knockout 4T1 cells}

To determine whether the effect of metformin on the efficacy of the TMV vaccine is due to the reduction of PD-L1, a 4T1 PD-L1 KO cell line was created using CRISPR/Cas9 

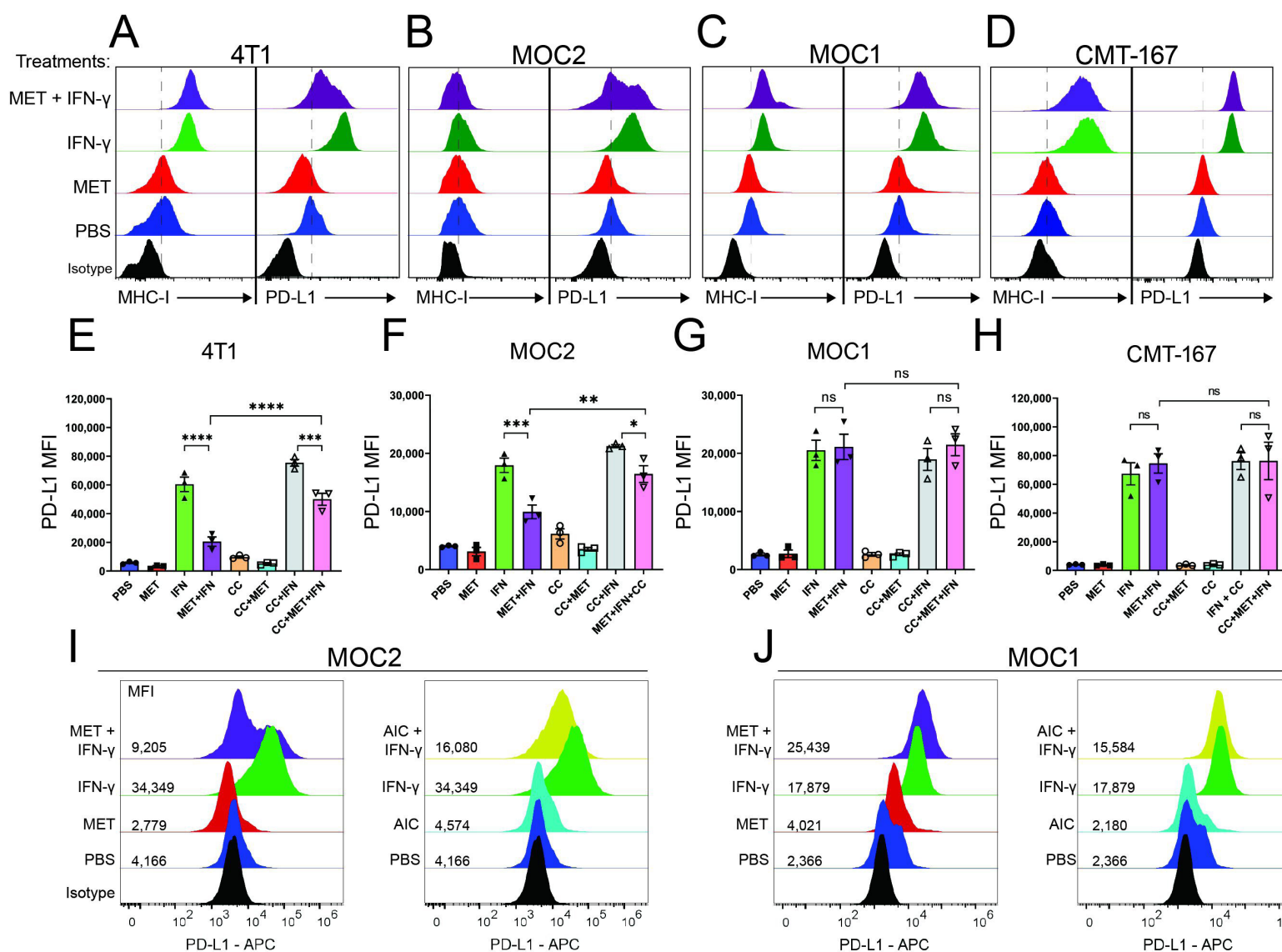

MOC2
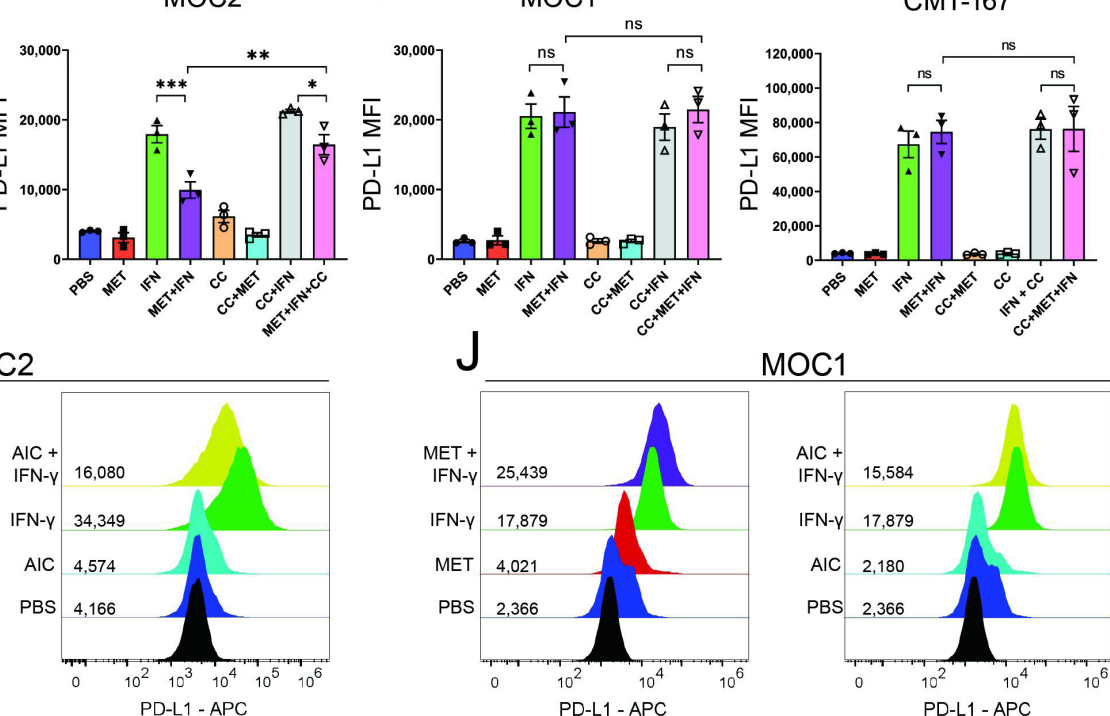

MOC1

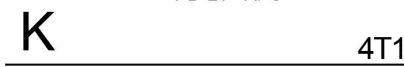

$\mathrm{L}$
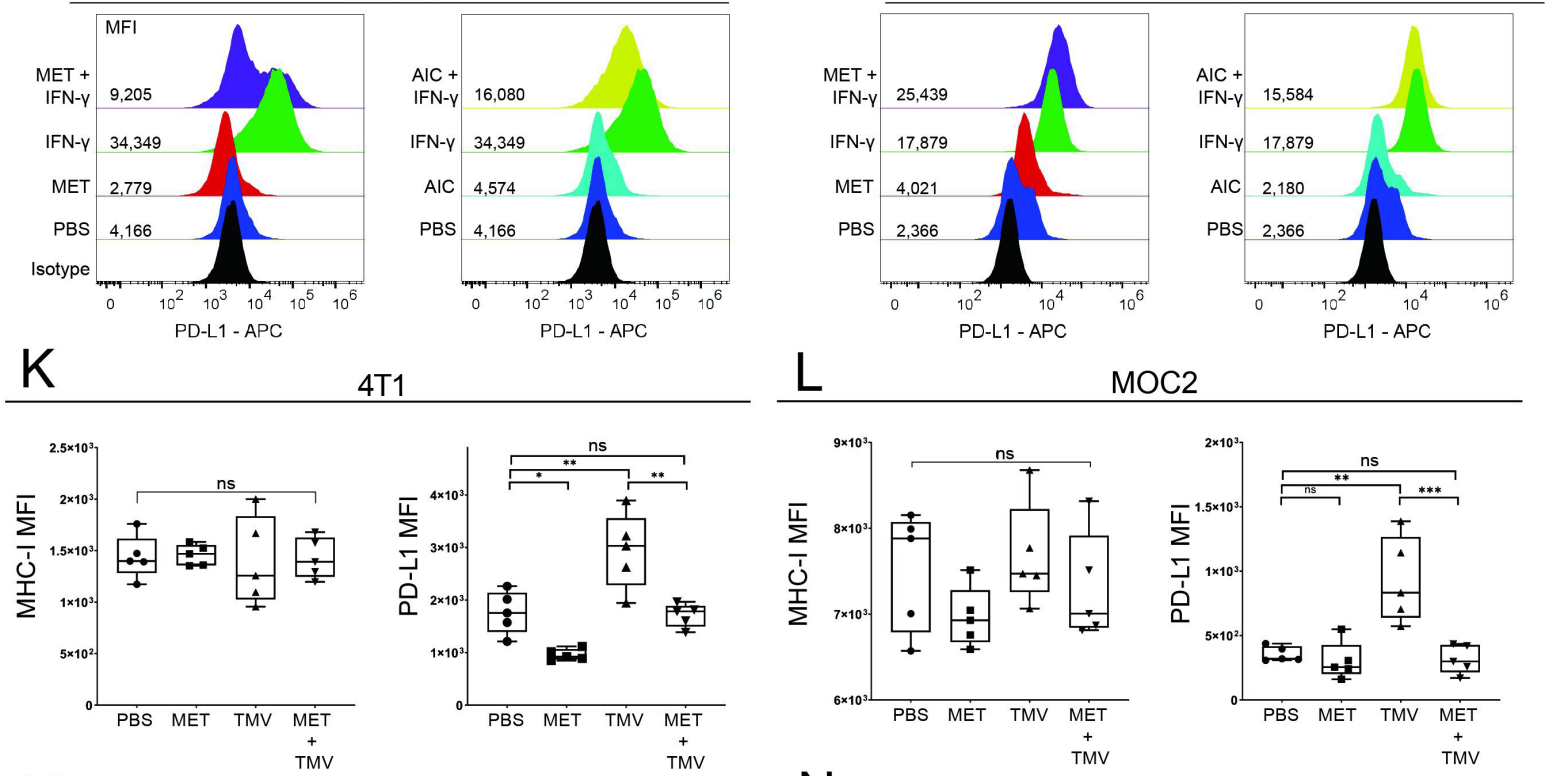

\section{M} CMT-167
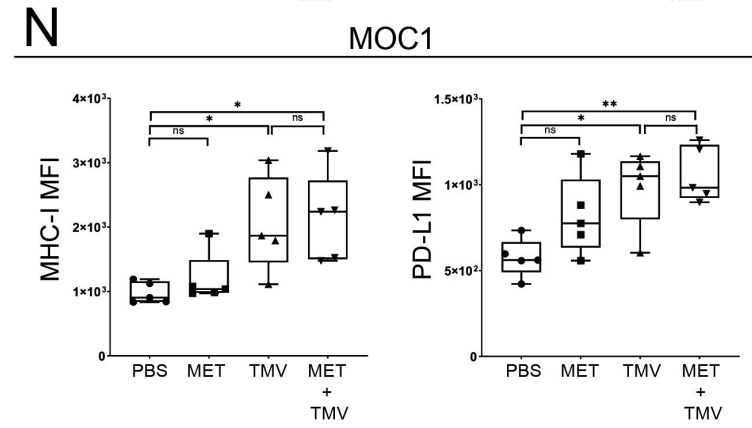

Figure 2 Metformin reduces surface PD-L1 partially through AMPK signaling. (A-D) Tumor cell lines were cultured in media with PBS as a control, $1 \mathrm{mM}$ metformin, $20 \mathrm{ng} / \mathrm{mL}$ of IFN- $\gamma$, or both for 48 hours and surface expression of relevant surface markers in vitro was determined by flow cytometry (black histograms represent isotype control from PBS treatment). (E-H) The cells plated as before, including the AMPK inhibitor Compound C (CC) at $5 \mu \mathrm{M}$ for 48 hours alone or in combination with metformin and IFN- $\gamma(\mathrm{n}=3$, mean \pm SEM). (l, J) MOC2 and MOC1 cell lines were cultured in media with $1 \mathrm{mM}$ metformin, $20 \mathrm{ng} / \mathrm{mL}$ IFN- $\gamma$, and/or the AMPK agonist $N^{1}$-( $\beta$-D-ribofuranosyl)-5-aminoimidazole-4-carboxamide (AICAR) at $100 \mu \mathrm{M}$ for 48 hours. (K-N) Tumors collected at days 25-30 postinoculation (as in Methods) and processed into single cell suspension to be analyzed by flow cytometry for surface expression of relevant markers on $\mathrm{CD}_{4} 5^{-}$cells $(\mathrm{n}=5$ mice per group). ( $\mathrm{E}-\mathrm{H})$ and $(\mathrm{K}-\mathrm{N})$ were analyzed using one-way analysis of variance (ANOVA) with Tukey's post test for multiple comparisons. TMV, tumor membrane vesicle. ${ }^{*} p<0.05,{ }^{* *} p<0.01,{ }^{* \star *} p<0.001,{ }^{* * * *} p<0.0001$, NS $p>0.05$. MOC1, murine oral carcinoma-1; MOC2, murine oral carcinoma-2. Additional data on AMPK in online supplemental figure S4. 
A
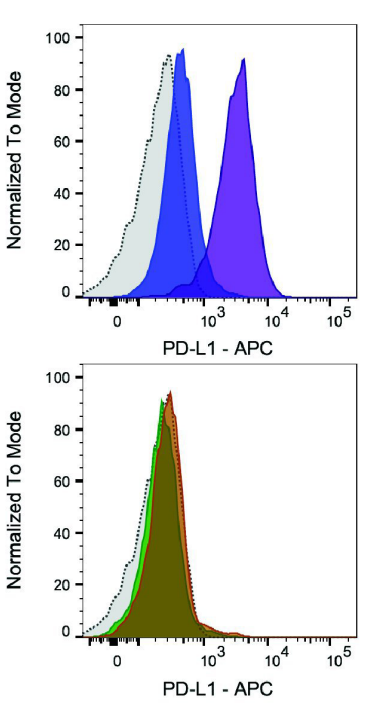
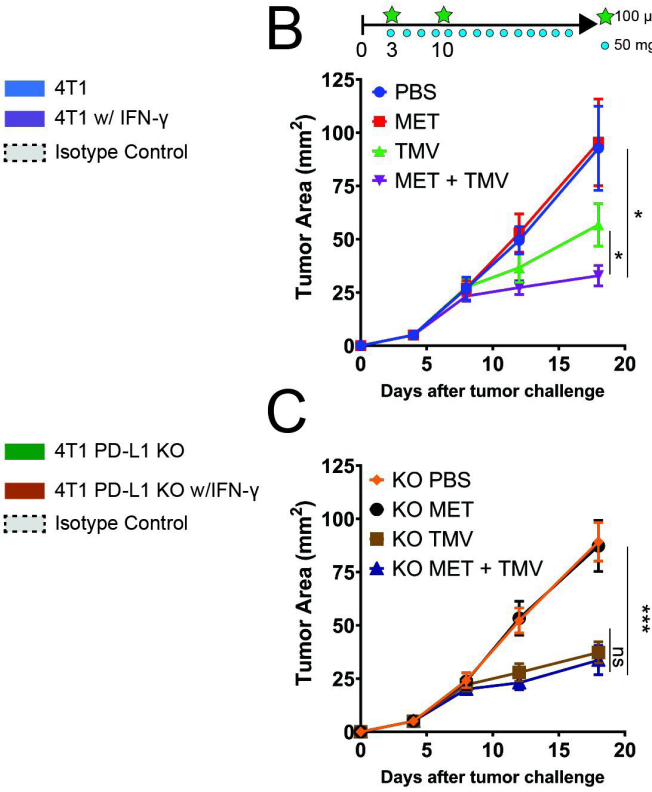

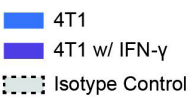
:.:.: Isotype Control

Figure 3 Metformin does not enhance tumor membrane vesicle (TMV) efficacy in PD-L1 KO 4T1 tumors. (A) PD-L1 histograms of $4 \mathrm{~T} 1$ and $4 \mathrm{~T} 1 \mathrm{PD}-\mathrm{L} 1 \mathrm{KO}$ cells cultured in vitro with IFN- $\gamma$ for 24 hours. (B) BALB/c mice were inoculated with 4T1 cells and treated as depicted on the diagram with $100 \mu \mathrm{g}$ TMV vaccine and/or $50 \mathrm{mg} / \mathrm{kg}$ metformin. (C) BALB/c mice were inoculated with 4T1 PD-L1 KO cells and treated as in $B(n=5$ mice per group, mean \pm SEM). Analysis in $B$ and $C$ done by a two-way analysis of variance (ANOVA) with Tukey's post test for multiple comparisons. ${ }^{*} p<0.05,{ }^{* * *} p<0.01, N S p>0.05$.

and two rounds of sorting as outlined in the methods section. The sorted 4T1 PD-L1 KO cells did not express PD-L1 on the surface at baseline or after IFN- $\gamma$ treatment confirming the efficiency of the knockout method (figure 3A). To determine if modulation of PD-L1 on tumor cells is essential for the improvement of the TMV vaccine efficacy we inoculated $\mathrm{BALB} / \mathrm{c}$ mice with $4 \mathrm{~T} 1$ or 4T1 PD-L1 KO cells. We found that there was a significant decrease in tumor growth after combination treatment compared with TMV vaccine alone in mice inoculated with 4T1 cells (figure 3B). In contrast, no difference in tumor growth was observed between TMV vaccine alone and the combination group in mice inoculated with PD-L1 KO 4T1 cells (figure 3C), suggesting that the efficacy of metformin is likely mediated in part by the modulation of PD-L1 on tumor cells.

\section{Metformin does not reduce PD-L1 expression in myeloid cells while TMV vaccination increases tumor-infiltrating DCs}

To determine how TMV vaccination and metformin might impact the immune milieu within tumors, we measured

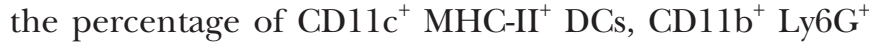
MDSCs, and $\mathrm{F} 4 / 80^{+}$tumor-associated macrophages (TAMs), as well as their expression of co-stimulatory and inhibitory markers. Both MOC2 and MOC1 had a significant increase in the percentage of $\mathrm{CD}_{11 \mathrm{c}^{+}} \mathrm{MHC}-$ $\mathrm{II}^{+}$DCs after TMV vaccination and this was not altered by metformin (figure 4B,H). These DCs also had a higher expression of surface CD86 and MHC-II in MOC2 (figure 4F,G) and MOC1 (figure 4L,M) models. Inversely, the percentage of MDSCs in the tumor was trending downward on TMV vaccination in MOC2 tumors, while combination with metformin resulted in a significant reduction of MDSCs compared with PBS controls (figure 4C). Similarly, in the MOC1 model, MDSCs were significantly reduced on TMV vaccination, while metformin had no measurable effect on the percentage of these cells (figure 4I). The percentage of macrophages within the tumor remains largely unchanged, with a slight trend downward, however this change was not statistically significant (figure 4D,J). Interestingly, in MOC1 tumors, TAMs had a significant increase in the expression of surface CD86 and MHC-II after therapy with TMV vaccine and metformin (figure $4 \mathrm{~L}, \mathrm{M}$ ). In contrast to tumor cells, PD-L1 was not reduced by metformin in DCs, MDSCs, and TAMs within MOC2 and MOC1 tumors (figure 4E,K).

\section{Metformin does not affect TMV vaccine induced T cell infiltration into tumors}

To understand the changes in the $\mathrm{T}$ cell compartment, FFPE sections of 4T1 tumors were used for the detection of CD3 T cells. While no changes were observed between the PBS and metformin treated tumors, TMV vaccine and the combination with metformin showed more CD3 $\mathrm{T}$ cells within the tumors (figure 5A,B). The distribution of CD3 T cells within 4T1 tumors was similar in both groups. We used flow cytometry to analyze the makeup of CD4 helper and CD8 cytotoxic T cells in resected tumors. We observed a significant increase of CD8 T cells within 4T1, CMT-167, MOC1 and MOC2 tumors from TMV vaccine treated mice (figure 5C-F). Metformin alone had no observable changes in the numbers of either subset of $\mathrm{T}$ cells across tumor models. Similar to the FFPE data, there was no difference between combination TMV vaccine and metformin treatment and TMV vaccine alone in the number of CD4 and CD8 cells. 


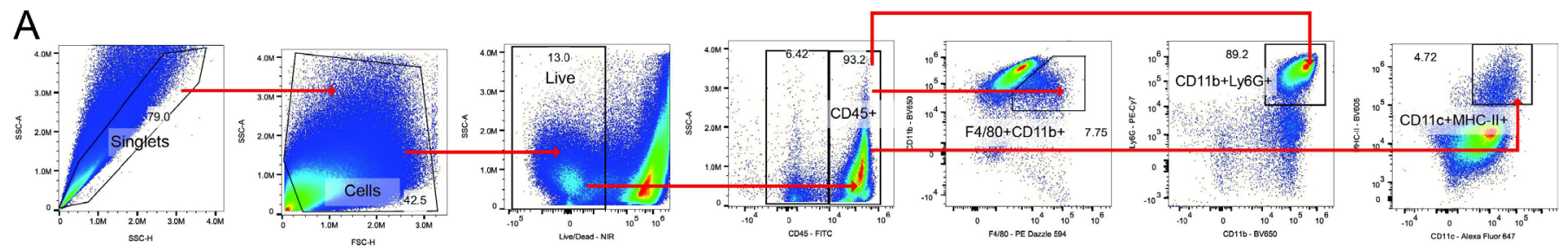

MOC2
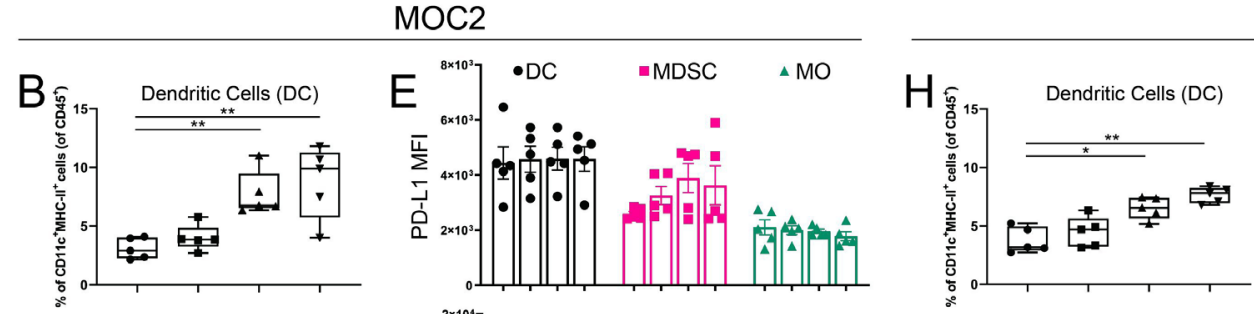

MOC1
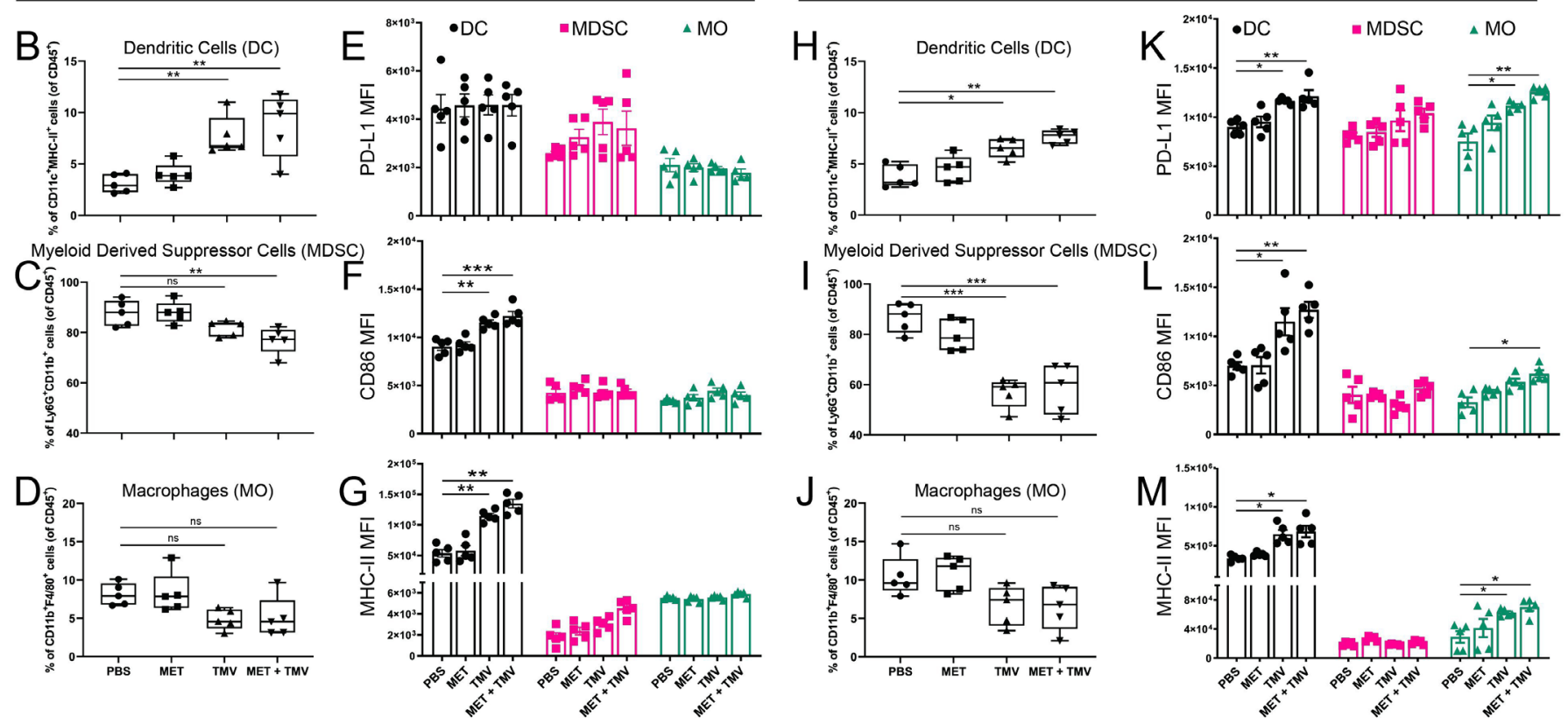

Figure 4 Metformin does not reduce PD-L1 expression on myeloid cells while tumor membrane vesicle (TMV) vaccine improves dendritic cell (DC) infiltration and activation. Cancer cells were inoculated in the hind flank and mice were treated as outlined in figure 2. MOC1 and MOC2 tumors were collected at days 25-30 postinoculation (according to Methods) and processed into single cell suspension to be analyzed by flow cytometry for surface expression of relevant markers. (A) Gating strategy for the three subsets. (B-D) and (H-J) represent the percentage of DCs, myeloid derived suppressor cells (MDSCs) and macrophages $(\mathrm{MO})$ within the $\mathrm{CD} 45^{+}$cells in the tumors. $(\mathrm{E}-\mathrm{G})$ and $(\mathrm{K}-\mathrm{M})$ represent the median fluorescence intensity (MFI) of the surface markers PD-L1, CD86, and MHC-II ( $n=5$ mice per group, mean \pm SEM). B-M analyzed using one-way analysis of variance (ANOVA) with Tukey's post test for multiple comparisons. MOC1, murine oral carcinoma-1; MOC2, murine oral carcinoma-2. ${ }^{*} p<0.05,{ }^{* *} p<0.01,{ }^{* * *} p<0.001$, NS $p>0.05$.

\section{Metformin alters the phenotype and PD-1 expression of vaccine generated CD8 T cells}

Since PD-1 is the natural receptor for PD-L1 and it is found mostly on activated/exhausted $\mathrm{T}$ cells within the tumor, we analyzed the PD-1 expressing tumor-infiltrating CD8 $\mathrm{T}$ cells in all four tumor models. The majority of CD8 T cells were PD- $1^{\text {hi }}$ at baseline in PBS and metformin groups of all tumor models, ranging from $60 \%$ to $80 \%$ (figure 6B-E). Metformin treatment alone showed no discernible effect on surface PD-1 expression within CD8 T cells. Interestingly, TMV vaccination resulted in a significant decrease in the percentage of these PD-1 expressing CD8 T cells across tumor models, making up around $20 \%-40 \%$ of CD8 T cells. After treatment with combination therapy, metformin-sensitive tumors (4T1 and MOC2) regained the percentage of PD-1 expressing CD8 $\mathrm{T}$ cells (figure 6A upper panels, B,C). Inversely, $\mathrm{CD} 8 \mathrm{~T}$ cells in those tumors that were not sensitive to metformin (CMT-167 and MOC1) retained the phenotype observed with TMV vaccination alone (figure 6A lower panels, D,E). To further elucidate the phenotype of these cells, we analyzed the tumor-infiltrating CD8 T cells in $4 \mathrm{~T} 1$ tumors after treatment. There was a significant increase in the percentage of $\mathrm{CD} 62 \mathrm{~L}^{+} \mathrm{CD} 44^{+}$cells in the combination group compared with TMV vaccine alone (figure $6 \mathrm{~F}, \mathrm{G}$ ) and inversely a significant decrease in the percentage of $\mathrm{CD} 62 \mathrm{~L}^{-} \mathrm{CD} 44^{+} \mathrm{CD} 8 \mathrm{~T}$ cells in the combination group (figure $6 \mathrm{~F}, \mathrm{H}$ ). Further analysis of the CD62L subset showed that metformin treatment results in increased percentages of short-lived KLRG- $1^{+}$effector CD8 $\mathrm{T}$ cells (figure 6I,J). Interestingly, the proliferation marker Ki-67, and the terminal effector marker Tim-3 were significantly increased in the combination group compared with TMV vaccine alone, while the co-stimulatory activation marker ICOS was unchanged across treatment groups (figure 6K-M).

\section{Metformin enhances cytokine production of vaccine generated CD8 $\mathrm{T}$ cells within tumors}

To determine if the metformin treatment alters the cytokine production capability of cytotoxic $\mathrm{T}$ cells within 4T1 tumors, we isolated CD8 $\mathrm{T}$ cells from tumors and 


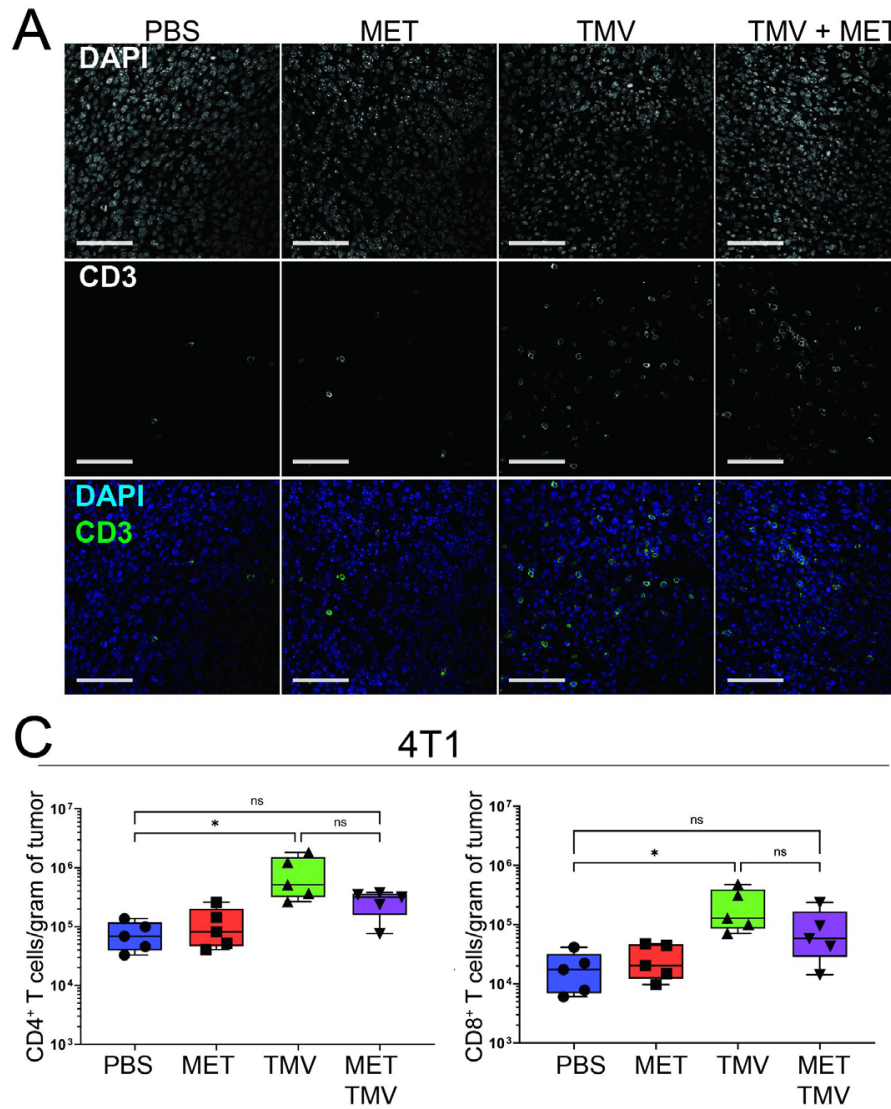

B
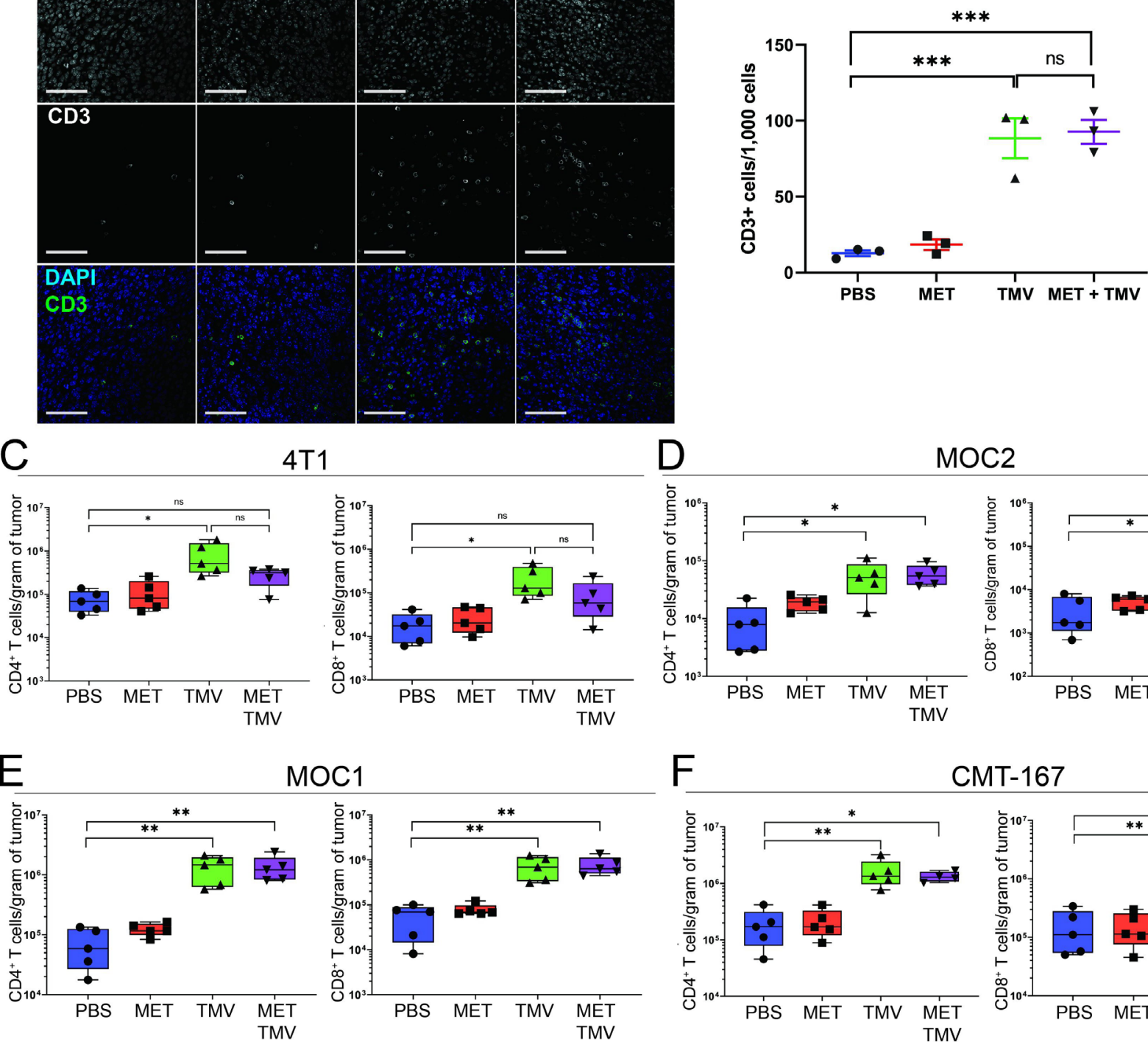

MOC1
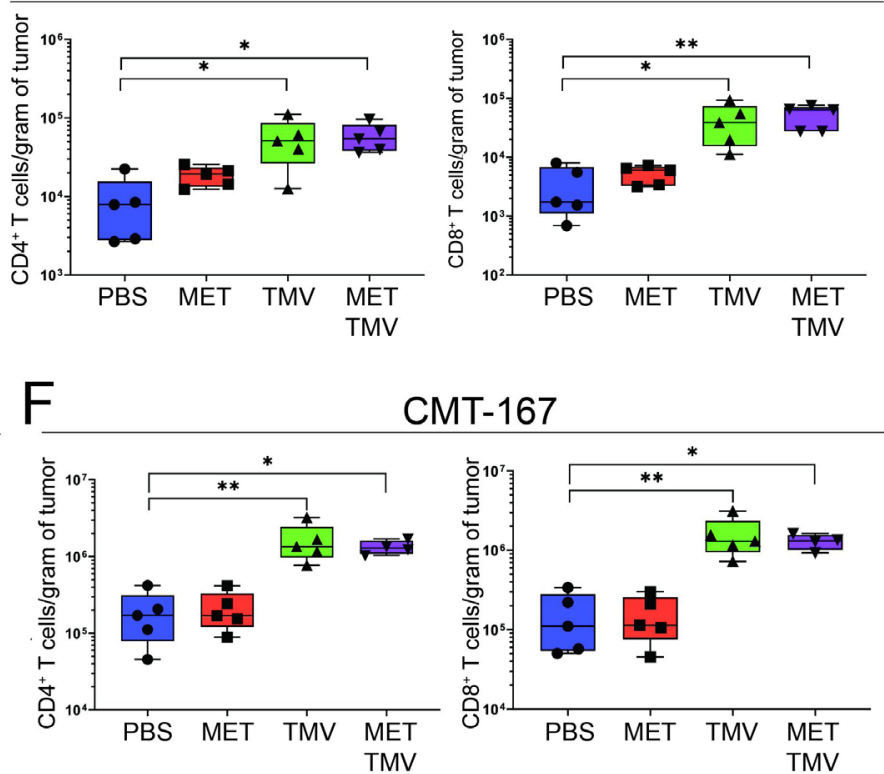

CMT-167
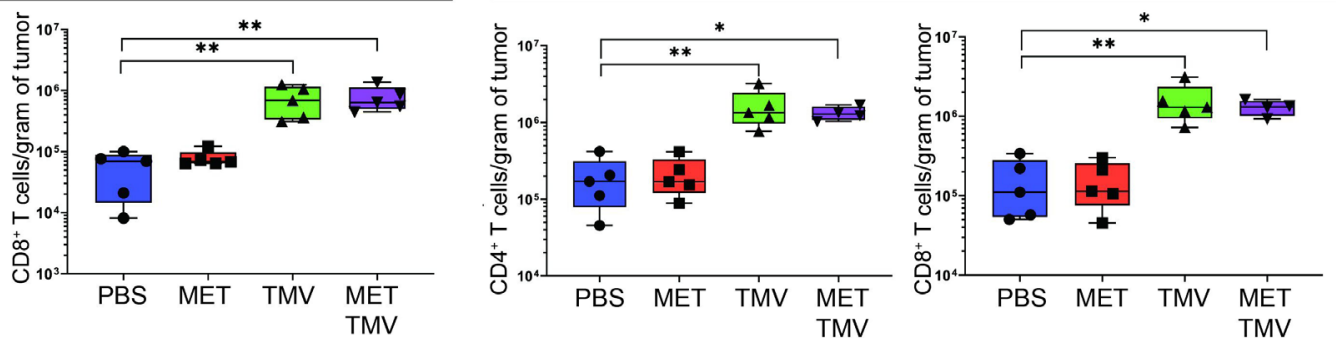

Figure 5 Tumor membrane vesicle (TMV) vaccination increases T cell infiltration into the tumor. (A) Formalin-fixed paraffinembedded (FFPE) sections from 4T1 tumors in figure 1A were stained for DAPI and CD3 and representative images are shown, scale bar represents $100 \mu \mathrm{m}$. (B) Quantification from A of CD3 T cells and overall DAPI cells using ImageJ ( $n=3$, mean $\pm S E M)$. (C-F) Tumors were harvested on days $25-30$ as described in Methods and prepared into single cell suspension; surface markers were analyzed using flow cytometry $(n=5)$. B-F analyzed by one-way analysis of variance (ANOVA) with Tukey's post test for multiple comparisons. MOC1, murine oral carcinoma-1; MOC2, murine oral carcinoma-2. ${ }^{\star} p<0.05,{ }^{\star \star} p<0.01,{ }^{* \star} p<0.001$, NS $\mathrm{p}>0.05$.

stimulated ex vivo with PMA/ionomycin to quantify IFN- $\gamma$, TNF- $\alpha$ and IL-2 producing cells (figure 7A) as described in the Methods section. The percentage of IFN- $\gamma$ and TNF- $\alpha$ double producers $\left(\mathrm{CD}^{+}\right.$IFN- $\gamma^{+}$TNF$\alpha^{+}$) increased significantly in the combination treatment group compared with PBS controls and TMV vaccine alone (figure 7B). Similarly, the percentage of IFN- $\gamma$ and IL-2 double producers $\left(\mathrm{CD}^{+}\right.$IFN- $\gamma^{+}$IL-2 $\left.{ }^{+}\right)$increased significantly in the combination group (figure $7 \mathrm{C}$ ). However, the percentage of TNF- $\alpha$ and IL-2 double producers remained similar in the TMV vaccine and combination treatment groups but higher than the PBS controls, but was not statistically significant (figure 7D). Overall, our results show that there is an increase in the percentage of cytokine-producing tumor-infiltrating CD8 T cells after TMV vaccine and metformin combination treatment.

\section{DISCUSSION}

Here we report on the use of metformin to increase the efficacy of a therapeutic cancer vaccine. We show that metformin reduced PD-L1 on tumors and this was partially dependent on AMPK signaling. This reduction in PD-L1 was seen at the protein level but not at the mRNA level, which is in agreement with a previous report. ${ }^{16}$ Using the TMV vaccine as a form of immunotherapy, we observed 
A Gated on Live CD $45^{+} \mathrm{CD} 3^{+} \mathrm{CD} 8^{+}$TILs

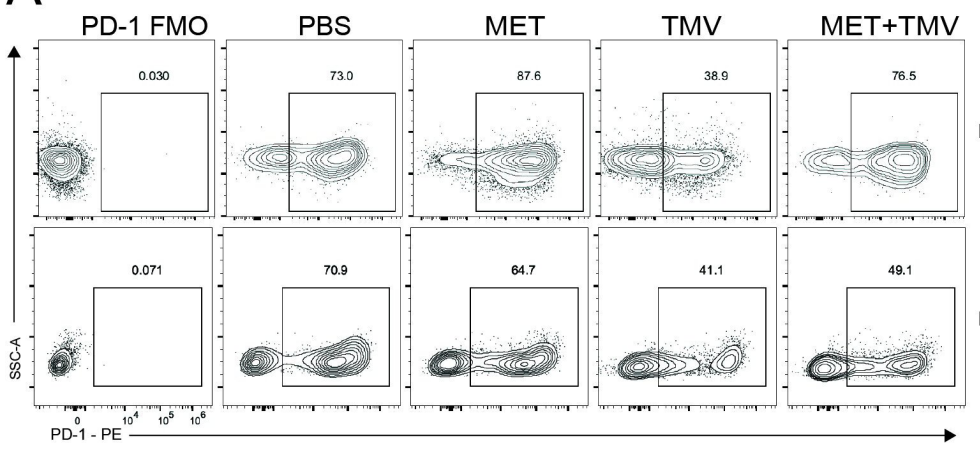

$\mathrm{F}$ Gated on $4 \mathrm{~T} 1$ Live $\mathrm{CD} 45^{+} \mathrm{CD}^{+} \mathrm{CD} 8^{+} \mathrm{TILs}$

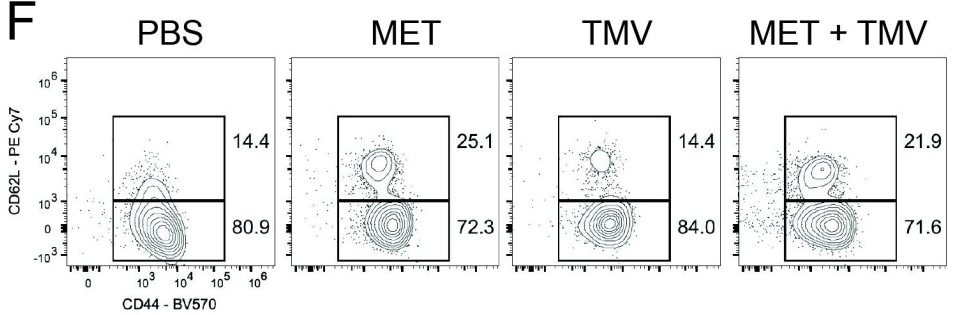

Gated on $4 \mathrm{~T} 1$ Live $\mathrm{CD} 45^{+} \mathrm{CD} 3^{+} \mathrm{CD} 8^{+} \mathrm{CD} 44^{+} \mathrm{CD} 62 \mathrm{~L}^{-}$cells
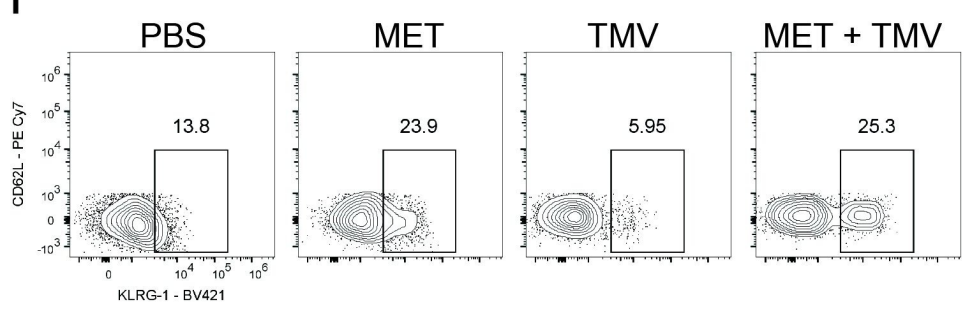

G
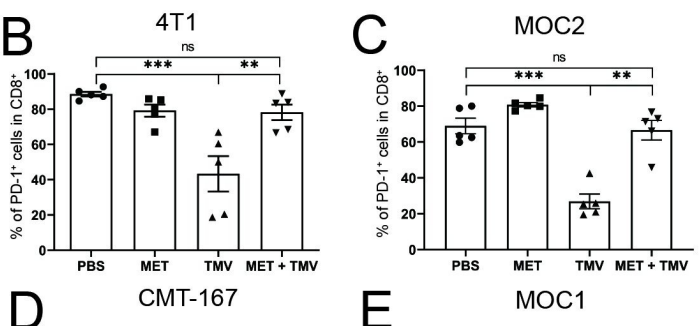

MOC1
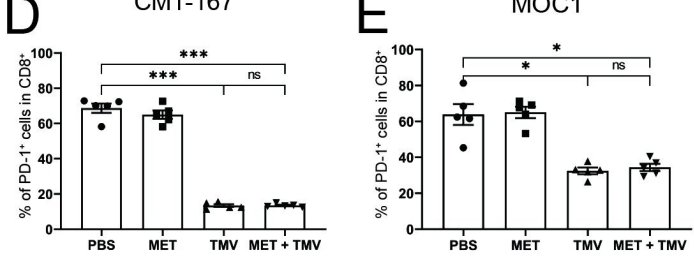

$\mathrm{H}$
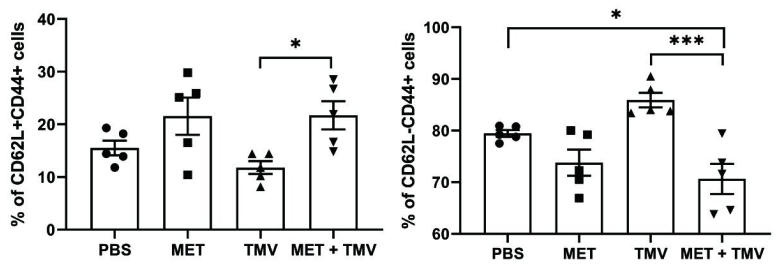

$\mathrm{J}$

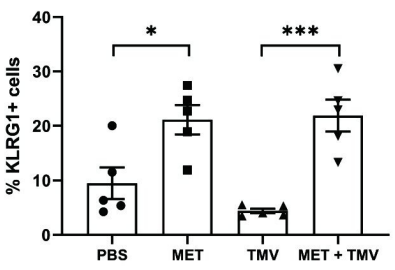

$\mathrm{K}$

Ki-67

$\mathrm{L}$

Tim-3

$\mathrm{M}$

ICOS
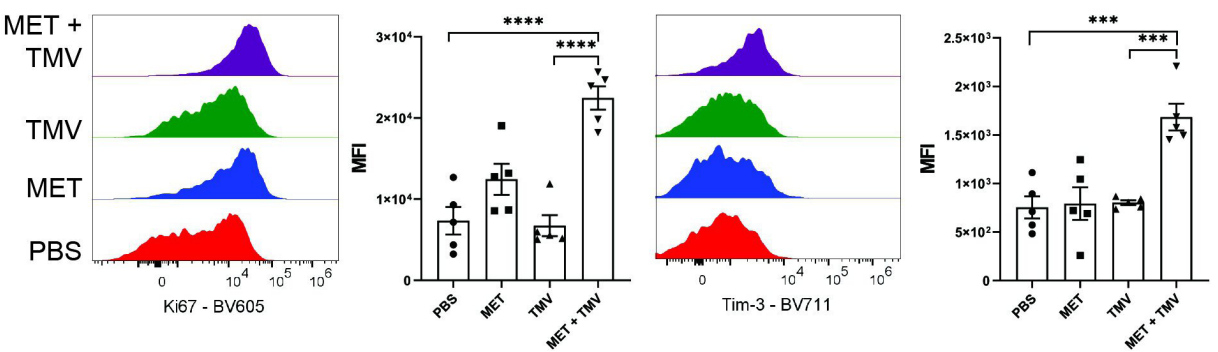

Figure 6 Metformin alters phenotype of tumor membrane vesicle (TMV) vaccine induced CD8 T cells in tumors. (A)

Representative contour plots of MOC2 and MOC1 PD-1 expression in CD8 T cell within the tumor with fluorescence minus one (FMO) controls. (B-E) Percentage of PD-1 expressing CD8 T cells in 4T1, CMT-167, MOC2, and MOC1 tumors 25-30 days after inoculation. (F) Representative contour plots of CD62L and CD44 expression in CD8 T cells within 4T1 tumors treated as in figure 1. $(\mathrm{G}, \mathrm{H})$ Percentage of central memory $\left(\mathrm{CD} 62 \mathrm{~L}^{+} \mathrm{CD} 44^{+}\right)$and effector/effector memory (CD62L-CD44 ${ }^{+}$) CD8 T cells from F. (I) Contour plots of CD62L-CD44 ${ }^{+}$CD8 T cells expressing KLRG-1 within 4T1 tumors. (J) Quantification of KLRG-1 $1^{+}$cells from I. (K-M) MFI expression of the nuclear factor and activation markers Ki-67, Tim-3 and ICOS on the surface of CD8 T cells within $4 T 1$ tumors $(n=5$, mean \pm SEM). B-M was analyzed using one-way analysis of variance (ANOVA) and Tukey's post test was used for multiple comparisons. MOC1, murine oral carcinoma-1; MOC2, murine oral carcinoma-2. ${ }^{*} p<0.05,{ }^{* *} p<0.01,{ }^{* * *} p<0.001$, ${ }^{\star * \star \star} p<0.0001$, NS $p>0.05$.

that metformin enhances the efficacy of TMV vaccine immunotherapy, but only when cell surface PD-L1 expression on tumors is downmodulated by metformin. The effect of metformin on the vaccine efficacy was lost in the PD-L1 KO 4T1 cell line further suggesting that downregulation of PD-L1 expression by metformin plays a role in enhancing the vaccine efficacy. Metformin also enhanced the expression of activation markers and cytokine production by tumor-infiltrating CD8 T cells.

AMPK is a major target of metformin due to its ability to inhibit complex I in the mitochondria. ${ }^{2122}$ Many mechanisms of action have been reported for metformin's antitumor effects, which can be tumor cell intrinsic or extrinsic. One reported mechanism is the reduction of 
A
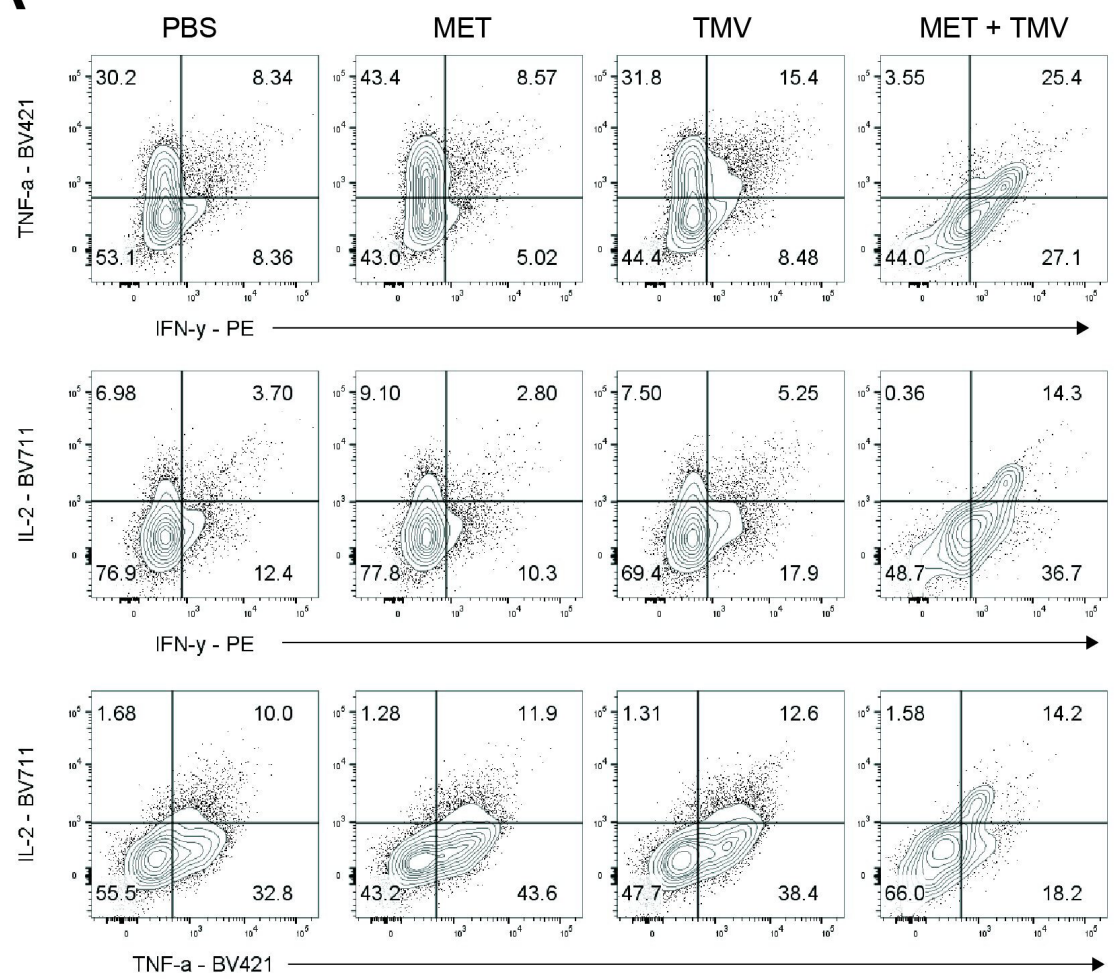
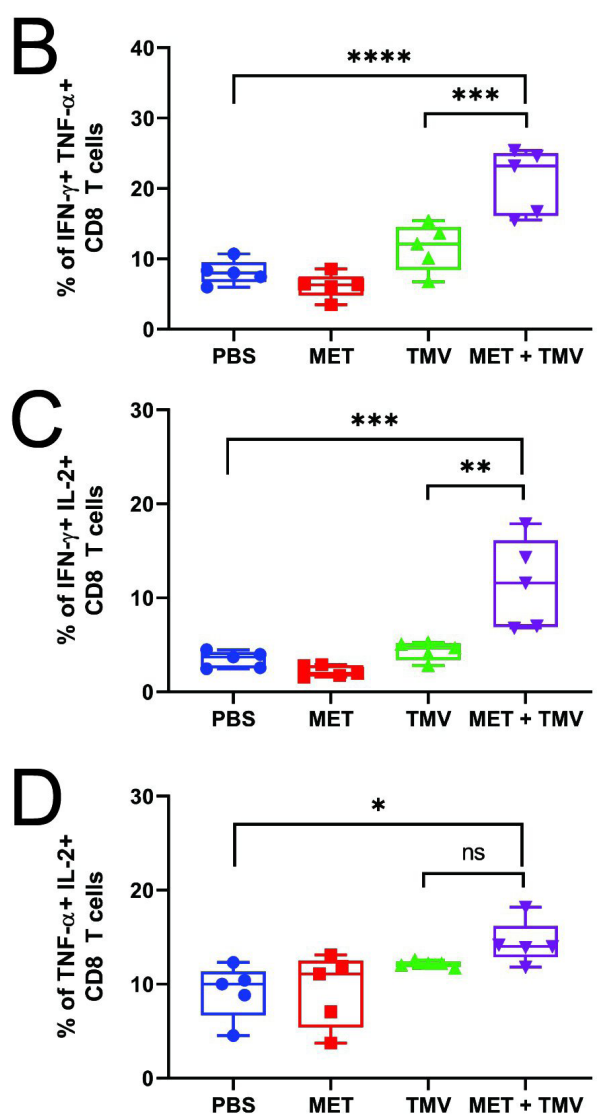

Figure 7 Metformin enhances double cytokine-producing vaccine-induced CD8 T cells within tumors. 4T1 tumors were harvested 25 days after inoculation and CD8 T cells were isolated as described in the Methods section. Cells were stimulated with PMA/ionomycin for 6 hours and cytokine producing cells were quantified by flow cytometry. (A) Contour plots of double cytokine producing CD8 T cells for IFN- $\gamma$, IL-2 and TNF- $\alpha$. (B) Quantification of IFN- $\gamma$ and TNF- $\alpha$ producing CD8 T cells. (C) Quantification of IFN- $\gamma$ and IL-2 producing CD8 T cells. (D) Quantification of TNF- $\alpha$ and IL-2 producing CD8 T cells $(n=5$ mice per group). Analysis in B-D done by a one-way analysis of variance (ANOVA) with Tukey's post test for multiple comparisons. TMV, tumor membrane vesicle. ${ }^{*} p<0.05,{ }^{* *} p<0.01,{ }^{* \star \star} p<0.01,{ }^{* \star \star *} p<0.001, N S p>0.05$.

tumor cell surface PD-L1 expression, which happens at the protein level from ER-associated degradation. ${ }^{16}$ After activation by metformin, AMPK directly phosphorylates PD-L1, resulting in abnormal glycosylation, and eventual degradation in the ER. In our study, the difference between metformin responsive and non-responsive models correlated with modulation of PD-L1 expression. Cell lines that had a reduction in surface PD-L1 expression on treatment with the AMPK activators metformin or AICAR in vitro also responded better to combination of TMV vaccine and metformin therapy. To test whether metformin acts via AMPK in downregulating PD-L1, we treated cells with the AMPK inhibitor Compound $\mathrm{C}$ and measured cell surface PD-L1. The results showed a partial restoration of PD-L1, suggesting that other signaling pathways in addition to AMPK are involved in the regulation of PD-L1 by metformin. Other reported pathways that downregulate PD-L1 include MYC, ${ }^{23}$ MAPK, ${ }^{24}$ and the Hippo pathway, ${ }^{25}{ }^{26}$ which are known to be affected by metformin. ${ }^{27}{ }^{28}$ We also observed, as others have, that the concentration of metformin required for activation of AMPK in vitro is much higher than the reported in vivo levels. ${ }^{29}$ While this could be due to the abundance of nutrients, growth factors and oxygen in culture, compared with the hypoxic and nutrient-deficient tumor microenvironment, it is likely that metformin is not the sole activator of AMPK in tumors in vivo.

To investigate whether metformin acts via mechanisms other than PD-L1 downregulation in the PD-1 resistant 4T1 model, we blocked the PD-1 pathway using an antiPD-1 monoclonal antibody $(\mathrm{mAb})$ and administered metformin. We found that the combination treatment suppressed tumor growth synergistically suggesting that metformin can also control tumor growth via mechanisms independent of the PD-1/PD-L1 pathway. Although our study does not address these other mechanisms, it has been reported that metformin can improve antitumor immunity beyond the PD-L1 pathway. One proposed mechanism is through the inhibition of mitochondrial complex I, which diminishes cellular respiration and reduces the hypoxic activation of HIF-1. ${ }^{21}$ This change in the tumor microenvironment by the reduction of tumor hypoxia improves $\mathrm{T}$ cell cytokine production and tumor clearance when combined with PD-1 blockade immunotherapy. ${ }^{18}$ Treatment of antigen-specific OT-I cells ex vivo with metformin results in improved cytokine production 
and reduced apoptosis of tumor-infiltrating CD8 T cells, ${ }^{30}$ which points to a $\mathrm{T}$ cell intrinsic effect as another mechanism of action for metformin.

CD8 T cells are not the only important immune cells within tumors, as the presence of DCs that create an antigen presenting niche in the tumor are essential to provide support for $\mathrm{T}$ cells. ${ }^{31}$ In the tumor models we tested, tumor-infiltrating $\mathrm{T}$ cells as well as DCs increased on TMV vaccination within MOC2 and MOC1 tumors (responsive and non-responsive, respectively). It remains to be seen if the phenotype and subsets of these DCs is affected by the administration of metformin. MDSCs are one of the major components of the tumor immune milieu and these can exert their suppressive effect by many mechanisms, including production of Arginase, ROS, cytokines, and expression of surface markers like PD-L1. ${ }^{32}$ Metformin did not influence the percentage of MDSCs or their expression of PD-L1, but this does not rule out the possibility of metformin reducing the suppressive capabilities of these cells as others have reported..$^{33}{ }^{34}$ Similarly, the percentage of macrophages and their surface PD-L1 expression was also largely unaffected by treatment with metformin.

PD-1 is the major receptor for PD-L1 and a primary regulator of $\mathrm{T}$ cell activation and exhaustion. We investigated the expression of PD-1 on tumor-infiltrating CD8 $\mathrm{T}$ cells, as these are known to engage with tumor cells directly. Metformin administered as monotherapy did not have any measurable effects on the percentage of PD-1 expressing CD8 T cells. However, administration of the TMV vaccine significantly decreased the percentage of PD-1 expressing CD8 T cells. It has been reported that IL-12 produced by DCs can reduce PD-1 expression in CD8 T cells after stimulation with a TLR9 agonist. ${ }^{35}$ The increase in tumor-infiltrating DCs by the TMV vaccine could have a similar effect in modulating PD-1 expression in CD8 T cells. In other cancer vaccine approaches, a decrease in PD-1 expressing tumor-infiltrating CD8 T cells has been observed, ${ }^{36}$ with a phenotype similar to what we observed in the TMV vaccine group. Importantly, in metformin responsive models, the percentage of PD-1 expressing CD8 $\mathrm{T}$ cells increased in the combination group compared with TMV vaccine alone, while this does not happen in non-responsive models.

In addition to PD-1 expression, we have also analyzed the effect of combination therapy on the phenotype of tumor-infiltrating CD8 T cells. As others have reported, metformin can increase the frequency of central memory and stem-cell memory CD8 T cells in the tumor, ${ }^{37}$ which have been shown to be beneficial in different tumor models. ${ }^{38}{ }^{39}$ A phenotypic shift towards $\mathrm{CD}_{62 \mathrm{~L}^{+}} \mathrm{CD} 44^{+}$ central memory CD8 T cells was observed in the combination group compared with TMV vaccine alone, which had an elevated proportion of CD62L CD44 ${ }^{+}$effector CD8 T cells. While these effector CD8 T cells have better cytotoxic capabilities, ${ }^{40}$ the central memory phenotype has better proliferative capabilities, as we observed an increase in $\mathrm{Ki}-67$ expression, leading to increased generation of antitumor immunity. Interestingly, metformin alone increased Ki- $67^{+}$CD8 $\mathrm{T}$ cells (although not statistically significant), but the increase was significant in combination with the TMV vaccine. However, treatment with the TMV vaccine alone resulted in a significant increase in tumor-infiltrating CD8 $\mathrm{T}$ cells in all the tumor models. One possible explanation for this outcome is that the TMV vaccine can increase the infiltration of vaccine-induced $T$ cell from the periphery into the tumor, while metformin can alter the tumor microenvironment and act on tumor infiltrating lymphocytes, leading to improved CD8 T cell functionality, proliferation and phenotype. These result in a synergistic effect of metformin and TMV vaccine in controlling tumor growth and metastasis in ICI-resistant 4T1 and MOC2 tumor models.

CD8 T cell functionality is another important parameter in quantifying the efficacy of the antitumor immune response. Decreased effector cytokine production by tumor infiltrating CD8 $\mathrm{T}$ cells is a hallmark of $\mathrm{T}$ cell exhaustion. ${ }^{41}$ TMV vaccine immunotherapy increased the percentage of IFN- $\gamma$ producing CD8 T cells in the tumor, whereas combination with metformin increased the percentage of cells producing IFN- $\gamma$ plus IL-2 or TNF- $\alpha$. Metformin alone has been reported to increase the production of these cytokines in CD8 $\mathrm{T}$ cells within the tumor, ${ }^{1830}$ however, we observed these changes only in combination with TMV vaccine immunotherapy. This is likely due to the suppressive and tumorigenic nature of 4T1 tumors compared with the ovalbumin-expressing immunogenic MO5 tumors used in the previous study. ${ }^{30}$

Clinically, metformin has generated mixed results as a therapeutic agent for the treatment of cancer. One clinical trial examining metformin as a treatment for nonmetastatic breast cancer showed a significant increase in progression-free survival (PFS) compared with placebo. ${ }^{42}$ In combination with chemotherapy, some clinical trials reported an increase in the overall response rate (ORR) with metformin co-administration. ${ }^{43}$ However, other trials do not show any changes in PFS or ORR, despite showing promising changes in tumor proliferation and apoptosis markers. ${ }^{44-46}$ Although most trials have focused on cancer prevention in patients with diabetes, many ongoing clinical trials are using metformin as a therapeutic agent for the treatment of breast, prostate, bladder, lung, skin, colorectal and renal cell carcinomas (ClinicalTrials. gov). While most of these studies focus on metformin as adjuvant or neo-adjuvant therapy with chemotherapy, radiotherapy, or checkpoint blockade, our data provide insight into the use of metformin as an adjuvant for vaccine immunotherapy. Finally, our results offer further understanding on how metformin modulates antitumor responses and suggest that differences in modulation of tumor-intrinsic factors can lead to different therapeutic outcomes after vaccine immunotherapy.

Twitter Luis Enrique Munoz @Luis_EMG_

Acknowledgements The authors thank Emory University Pediatrics and Winship Flow Cytometry core, the Winship Cancer Tissue and Pathology core, the Emory 
Integrated Cellular and Imaging core for their services. The authors also thank Drs Haydn Kissick and Rabindra Tirouvanziam from Emory University for feedback and critical reading of the manuscript.

Contributors LEM, RB, MS and PS designed experiments. LEM, LH, RB, RS, RNG, LM and SGS performed experiments. CDP, SR, SJCR provided key reagents. LEM, $\mathrm{RB}, \mathrm{MS}$ and PS interpreted data. LEM, RB and PS wrote the manuscript. PS is the guarantor of the manuscript.

Funding This work was supported by NCI/NIH R01 grants R01 CA202763 to PS and CDP, and R01CA208328to MS, and a Diversity Supplement R01 CA202763-S to LEM.

Competing interests PS holds shares in Metaclipse Therapeutics Corporation, a company that is planning to use GPI-anchored molecules to develop membranebased cancer vaccine in the future as suggested in the current manuscript.

\section{Patient consent for publication Not applicable.}

Ethics approval Experiments were done in accordance with Emory University Institutional Animal Care and Use Committee (IACUC) approved protocols (DAR-2017-00-504).

Provenance and peer review Not commissioned; externally peer reviewed.

Data availability statement Data are available upon reasonable request. Data and materials published are available under reasonable request.

Supplemental material This content has been supplied by the author(s). It has not been vetted by BMJ Publishing Group Limited (BMJ) and may not have been peer-reviewed. Any opinions or recommendations discussed are solely those of the author(s) and are not endorsed by BMJ. BMJ disclaims all liability and responsibility arising from any reliance placed on the content. Where the content includes any translated material, BMJ does not warrant the accuracy and reliability of the translations (including but not limited to local regulations, clinical guidelines, terminology, drug names and drug dosages), and is not responsible for any error and/or omissions arising from translation and adaptation or otherwise.

Open access This is an open access article distributed in accordance with the Creative Commons Attribution Non Commercial (CC BY-NC 4.0) license, which permits others to distribute, remix, adapt, build upon this work non-commercially, and license their derivative works on different terms, provided the original work is properly cited, appropriate credit is given, any changes made indicated, and the use is non-commercial. See http://creativecommons.org/licenses/by-nc/4.0/.

\section{ORCID iDs}

Rohini N. Guin http://orcid.org/0000-0002-

Periasamy Selvaraj http://orcid.org/0000-0003-3130-0551

\section{REFERENCES}

1 Ventola CL. Cancer immunotherapy, part 3: challenges and future trends 2017;42:514-21.

2 Hegde PS, Chen DS. Top 10 challenges in cancer immunotherapy. Immunity 2020;52:17-35.

3 Seidel JA, Otsuka A, Kabashima K. Anti-PD-1 and anti-CTLA-4 therapies in cancer: mechanisms of action, efficacy, and limitations. Front Oncol 2018;8:86.

4 Chae YK, Arya A, lams W, et al. Current landscape and future of dual anti-CTLA4 and PD-1/PD-L1 blockade immunotherapy in cancer; lessons learned from clinical trials with melanoma and non-small cell lung cancer (NSCLC). J Immunother Cancer 2018;6:39.

5 Haslam A, Prasad V. Estimation of the percentage of US patients with cancer who are eligible for and respond to checkpoint inhibitor immunotherapy drugs. JAMA Netw Open 2019;2:e192535.

6 Kamphorst AO, Pillai RN, Yang S, et al. Proliferation of PD-1+ CD8 T cells in peripheral blood after PD-1-targeted therapy in lung cancer patients. Proc Natl Acad Sci U S A 2017;114:4993-8.

7 Krieg C, Nowicka M, Guglietta S, et al. High-dimensional single-cell analysis predicts response to anti-PD-1 immunotherapy. Nat Med 2018;24:144-53.

8 Poloso NJ, Nagarajan S, Bumgarner GW, et al. Development of therapeutic vaccines by direct modification of cell membranes from surgically removed human tumor tissue with immunostimulatory molecules. Vaccine 2001;19:2029-38.

9 Patel JM, Vartabedian VF, Bozeman EN, et al. Plasma membrane vesicles decorated with glycolipid-anchored antigens and adjuvants via protein transfer as an antigen delivery platform for inhibition of tumor growth. Biomaterials 2016;74:231-44.
10 Nagarajan S, Selvaraj P. Human tumor membrane vesicles modified to express glycolipid-anchored IL-12 by protein transfer induce T cell proliferation in vitro: a potential approach for local delivery of cytokines during vaccination. Vaccine 2006;24:2264-74.

11 McHugh RS, Nagarajan S, Wang YC, et al. Protein transfer of glycosyl-phosphatidylinositol-B7-1 into tumor cell membranes: a novel approach to tumor immunotherapy. Cancer Res 1999;59:2433-7.

12 Pack CD, Bommireddy R, Munoz LE, et al. Tumor membranebased vaccine immunotherapy in combination with anti-CTLA-4 antibody confers protection against immune checkpoint resistant murine triple-negative breast cancer. Hum Vaccin Immunother 2020;16:3184-93.

13 Bommireddy R, Munoz LE, Kumari A, et al. Tumor membrane vesicle vaccine augments the efficacy of anti-PD1 antibody in immune checkpoint inhibitor-resistant squamous cell carcinoma models of head and neck cancer. Vaccines 2020;8. doi:10.3390/ vaccines8020182. [Epub ahead of print: 1404 2020].

14 Verdura S, Cuyàs E, Martin-Castillo B, et al. Metformin as an archetype immuno-metabolic adjuvant for cancer immunotherapy. Oncoimmunology 2019;8:e1633235.

15 Rena G, Hardie DG, Pearson ER. The mechanisms of action of metformin. Diabetologia 2017;60:1577-85.

16 Cha J-H, Yang W-H, Xia W, et al. Metformin promotes antitumor immunity via endoplasmic-reticulum-associated degradation of PDL1. Mol Cell 2018;71:606-20.

17 Han Y, Li C-W, Hsu J-M, et al. Metformin reverses PARP inhibitorsinduced epithelial-mesenchymal transition and PD-L1 upregulation in triple-negative breast cancer. Am J Cancer Res 2019;9:800-15.

18 Scharping NE, Menk AV, Whetstone RD, et al. Efficacy of PD-1 blockade is potentiated by metformin-induced reduction of tumor hypoxia. Cancer Immunol Res 2017;5:9-16.

19 Juneja VR, McGuire KA, Manguso RT, et al. Pd-L1 on tumor cells is sufficient for immune evasion in immunogenic tumors and inhibits CD8 T cell cytotoxicity. J Exp Med 2017;214:895-904.

20 Kim K, Skora AD, Li Z, et al. Eradication of metastatic mouse cancers resistant to immune checkpoint blockade by suppression of myeloidderived cells. Proc Natl Acad Sci U S A 2014;111:11774-9.

21 Wheaton WW, Weinberg SE, Hamanaka RB, et al. Metformin inhibits mitochondrial complex I of cancer cells to reduce tumorigenesis. Elife 2014;3:e02242.

22 El-Mir MY, Nogueira V, Fontaine E, et al. Dimethylbiguanide inhibits cell respiration via an indirect effect targeted on the respiratory chain complex I. J Biol Chem 2000;275:223-8.

23 Zou J, Zhuang M, Yu X, et al. Myc inhibition increases PD-L1 expression induced by IFN- $\gamma$ in hepatocellular carcinoma cells. $\mathrm{Mol}$ Immunol 2018;101:203-9.

24 Atefi M, Avramis E, Lassen A, et al. Effects of MAPK and PI3K pathways on PD-L1 expression in melanoma. Clin Cancer Res 2014;20:3446-57.

25 Zhang J-J, Zhang Q-S, Li Z-Q, et al. Metformin attenuates PD-L1 expression through activating Hippo signaling pathway in colorectal cancer cells. Am J Trans/ Res 2019;11:6965-76.

26 Hajimoradi Javarsiani M, Sajedianfard J, Haghjooy Javanmard S. The effects of metformin on the Hippo pathway in the proliferation of melanoma cancer cells: a preclinical study. Arch Physiol Biochem 2020:1-6.

27 Wang SS, Hsiao R, Limpar MM, et al. Destabilization of MYC/MYCN by the mitochondrial inhibitors, metaiodobenzylguanidine, metformin and phenformin. Int J Mol Med 2014;33:35-42.

$28 \mathrm{Wu} \mathrm{N}, \mathrm{Gu}$ C, Gu H, et al. Metformin induces apoptosis of lung cancer cells through activating JNK/p38 MAPK pathway and GADD153. Neoplasma 2011;58:482-90.

29 Dowling RJO, Lam S, Bassi C, et al. Metformin pharmacokinetics in mouse tumors: implications for human therapy. Cell Metab 2016;23:567-8

30 Eikawa S, Nishida M, Mizukami S, et al. Immune-mediated antitumor effect by type 2 diabetes drug, metformin. Proc Natl Acad Sci U S A 2015;112:1809-14.

31 Jansen CS, Prokhnevska N, Master VA, et al. An intra-tumoral niche maintains and differentiates stem-like CD8 T cells. Nature 2019;576:465-70.

32 Weber R, Fleming V, Hu X, et al. Myeloid-derived suppressor cells hinder the anti-cancer activity of immune checkpoint inhibitors. Front Immunol 2018;9:1310.

33 Li L, Wang L, Li J, et al. Metformin-Induced reduction of CD39 and CD73 blocks myeloid-derived suppressor cell activity in patients with ovarian cancer. Cancer Res 2018;78:1779-91.

34 Qin G, Lian J, Huang L, et al. Metformin blocks myeloid-derived suppressor cell accumulation through AMPK-DACH1-CXCL1 axis. Oncoimmunology 2018;7:e1442167. 
35 Yin P, Liu X, Mansfield AS, et al. CpG-induced antitumor immunity requires IL-12 in expansion of effector cells and down-regulation of PD-1. Oncotarget 2016;7:70223-31.

36 Gleisner MA, Pereda C, Tittarelli A, et al. A heat-shocked melanoma cell lysate vaccine enhances tumor infiltration by prototypic effector T cells inhibiting tumor growth. J Immunother Cancer 2020;8:e000999.

37 Zhang Z, Li F, Tian Y, et al. Metformin Enhances the Antitumor Activity of $\mathrm{CD}^{+} \mathrm{T}$ Lymphocytes via the AMPK-miR-107-Eomes-PD-1 Pathway. J Immunol 2020;204:ji1901213:2575-88.

38 Klebanoff CA, Gattinoni L, Torabi-Parizi P, et al. Central memory self/ tumor-reactive CD8+ T cells confer superior antitumor immunity compared with effector memory T cells. Proc Natl Acad Sci U S A 2005;102:9571-6.

39 Gattinoni L, Lugli E, Ji Y, et al. A human memory T cell subset with stem cell-like properties. Nat Med 2011;17:1290-7.

40 Wherry EJ, Teichgräber V, Becker TC, et al. Lineage relationship and protective immunity of memory CD8 T cell subsets. Nat Immunol 2003;4:225-34.
41 Wherry EJ, Kurachi M. Molecular and cellular insights into T cell exhaustion. Nat Rev Immunol 2015;15:486-99.

42 Saraei P, Asadi I, Kakar MA, et al. The beneficial effects of metformin on cancer prevention and therapy: a comprehensive review of recent advances. Cancer Manag Res 2019;11:3295-313.

43 Coyle C, Cafferty FH, Vale C, et al. Metformin as an adjuvant treatment for cancer: a systematic review and meta-analysis. Ann Oncol 2016;27:2184-95.

44 Schuler KM, Rambally BS, DiFurio MJ, et al. Antiproliferative and metabolic effects of metformin in a preoperative window clinical trial for endometrial cancer. Cancer Med 2015;4:161-73.

45 Mitsuhashi A, Kiyokawa T, Sato Y, et al. Effects of metformin on endometrial cancer cell growth in vivo: a preoperative prospective trial. Cancer 2014;120:2986-95.

46 Laskov I, Drudi L, Beauchamp M-C, et al. Anti-diabetic doses of metformin decrease proliferation markers in tumors of patients with endometrial cancer. Gynecol Oncol 2014;134:607-14. 